\title{
An implicit midpoint difference scheme for the fractional Ginzburg-Landau equation
}

\author{
Pengde Wang, Chengming Huang* \\ School of Mathematics and Statistics, Huazhong University of Science and Technology, Wuhan 430074, China
}

\begin{abstract}
This paper proposes and analyzes an efficient difference scheme for the nonlinear complex Ginzburg-Landau equation involving fractional Laplacian. The scheme is based on the implicit midpoint rule for the temporal discretization and a weighted and shifted Grünwald difference operator for the spatial fractional Laplacian. By virtue of a careful analysis of the difference operator, some useful inequalities with respect to suitable fractional Sobolev norms are established. Then the numerical solution is shown to be bounded, and convergent in the $l_{h}^{2}$ norm with the optimal order $O\left(\tau^{2}+h^{2}\right)$ with time step $\tau$ and mesh size $h$. The a priori bound as well as the convergence order hold unconditionally, in the sense that no restriction on the time step $\tau$ in terms of the mesh size $h$ needs to be assumed. Numerical tests are performed to validate the theoretical results and effectiveness of the scheme.
\end{abstract}

Key words: Fractional Ginzburg-Landau equation; Fractional Laplacian; Riesz fractional derivative; Weighted and shifted Grünwald difference; Convergence

\section{Introduction}

The classical complex Ginzburg-Landau equation (GLE) is one of the most-studied nonlinear equations in the physics community, which describes a vast variety of phenomena from nonlinear waves to second-order phase transitions, from superconductivity, superfluidity, and Bose-Einstein condensation to liquid crystals and strings in field theory [1]. The fractional generalization of the GLE was suggested in [2, 3] from the variational Euler-Lagrange equation for fractal media. Since then, the fractional Ginzburg-Landau equation (FGLE) has been exploited to describe various physical phenomena, such as the dynamical processes in continuums with fractal dispersion and the media with fractal mass dimension [2], a fairly general class of critical phenomena when the organization of the system near the phase transition point is influenced by a competing nonlocal ordering [4] and a network of diffusively Hindmarsh-Rose neurons with long-range synaptic coupling [5].

\footnotetext{
${ }^{*}$ Corresponding author. Email addresses: pengde_wang@yeah.net (P. Wang), chengming_huang@hotmail . com (C. Huang)
} 
In this paper, we consider the following FGLE with the fractional Laplacian $(1<\alpha \leqslant 2)$

$$
u_{t}+(v+i \eta)(-\Delta)^{\frac{\alpha}{2}} u+(\kappa+i \zeta)|u|^{2} u-\gamma u=0, \quad x \in \mathbb{R}, t \in(0, T]
$$

subject to the initial condition

$$
u(x, 0)=u_{0}(x), \quad x \in \mathbb{R},
$$

where $i=\sqrt{-1}, u(x, t)$ is a complex-valued function of time $t$ and space $x, v>0, \kappa>0, \eta, \zeta, \gamma$ are given real constants and $u_{0}(x)$ is a given smooth function. The fractional Laplacian can be regarded as a pseudo-differential operator with the symbol $-|\xi|^{\alpha}$ :

$$
-(-\Delta)^{\frac{\alpha}{2}} u(x, t)=-\mathscr{F}^{-1}\left(|\xi|^{\alpha} \hat{u}(\xi, t)\right),
$$

where $\mathscr{F}$ denotes the Fourier transform. It is indeed equivalent to the following Riesz fractional derivative [6, 7], i.e.,

$$
-(-\Delta)^{\frac{\alpha}{2}} u(x, t)=\frac{\partial^{\alpha}}{\partial|x|^{\alpha}} u(x, t):=-\frac{1}{2 \cos \frac{\alpha \pi}{2}}\left[{ }_{-\infty} D_{x}^{\alpha} u(x, t)+{ }_{x} D_{+\infty}^{\alpha} u(x, t)\right],
$$

where ${ }_{-\infty} D_{x}^{\alpha} u(x, t)$ denotes the left Riemann-Liouville fractional derivative [ [8]

$$
{ }_{-\infty} D_{x}^{\alpha} u(x, t)=\frac{1}{\Gamma(2-\alpha)} \frac{d^{2}}{d x^{2}} \int_{-\infty}^{x} \frac{u(\xi, t)}{(x-\xi)^{\alpha-1}} d \xi
$$

and ${ }_{x} D_{+\infty}^{\alpha} u(x, t)$ the right Riemann-Liouville fractional derivative

$$
{ }_{x} D_{+\infty}^{\alpha} u(x, t)=\frac{1}{\Gamma(2-\alpha)} \frac{d^{2}}{d x^{2}} \int_{x}^{+\infty} \frac{u(\xi, t)}{(\xi-x)^{\alpha-1}} d \xi .
$$

Obviously, when $\alpha=2$, this operator reduces to the classical Laplacian and the equation reduces to the classical cubic nonlinear complex GLE.

From the mathematical point of view, unlike the case of classical GLE, the dissipative mechanism of the FGLE is not characterized by the classical Laplacian but by the fractional power of the Laplacian, which has raised some essential difficulties in theoretical analysis and recently drawn quite a lot of interest from various authors. For example, Tarasov [9] derived and analyzed the psiseries solution. Pu and Guo [10] investigated the global well-posedness, long-time dynamics and global attractors for the nonlinear FGLE. Guo ang Huo [11] studied the inviscid limit behavior of the FGLE to the fractional Schrödinger equation (FSE). Lu et al. [12] analyzed the well-posedness and asymptotic behaviors in two dimensions. Millot and Sire [13] considered the asymptotic analysis of the FGLE in bounded domain and showed that solutions with uniformly bounded energy converge weakly to sphere valued 1/2-harmonic maps.

From the numerical point of view, however, there is very little attention to the numerical solution of the FGLE. In order to simulate the propagation of the localized impulses in diffuse neural networks, Mvogo et al. [5] proposed a semi-implicit Riesz fractional finite difference scheme, which is first order in time and second order in space. Numerical simulations show that the scheme 
is feasible and efficient. To the best of our knowledge, it seems that this is the only work. Nevertheless, that paper focused on the establishment and simulation of the model equation. The stability and convergence of the discretization scheme were not discussed.

In this paper, we propose another difference scheme for the FGLE, which treats the time derivative by the implicit midpoint rule and the space derivative by the second-order accurate weighted and shifted Grünwald difference (WSGD) method [14]. This scheme is second-order in both time and space. Our focus is on a rigorous theoretical analysis for the scheme. We will prove that the scheme is unconditionally convergent with optimal order, in the sense that no added restriction on the temporal step size in terms of the spatial discretization parameter needs to be assumed. We mention that there are a number of convergence results for the classical GLE in the literature (see, e.g., [15, 16, 17, 18, 19, 20, 21, 22] and reference therein), which are normally derived based on the uniform boundedness of numerical solutions [15, 16]. In our case, however, it seems difficult for us to follow this approach because of the nonlocal property of the fractional Laplacian. In order to overcome this obstacle, we make a detailed study of the fractional approximation operator. The discrete fractional Gagliardo-Nirenberg inequality and an equivalence relation between an energy norm and the fractional Sobolev semi-norm are established.

The remainder of this paper is arranged as follows. In Section 2, we introduce the WSGD operator to discretize the involved fractional derivative, and give some technical lemmas. In Section 3 , we establish our fully discrete scheme. Section 4 is devoted to the rigorous theoretical analysis, including unique solvability and especially, the boundedness and convergence. In Section 5, we carry out some numerical experiments to confirm our theoretical results and show the efficiency of the proposed scheme. Finally, we draw some conclusions in Section 6.

\section{Preliminaries}

\subsection{Spatial discretization}

Up to now, a broad range of difference methods have been constructed to approximate the Riemann-Liouville fractional derivative and Riesz fractional derivative, such as the Grünwaldbased scheme [23, 24, 25, 26, 14, 27, 28, 29, 30] and the fractional centered difference based scheme [31, 32, 33, 34]. Each of them has its own advantages.

In this paper, we use the WSGD method, proposed by Hao, Sun and Cao [14], to approximate the left and right Riemann-Liouville space fractional derivatives, respectively. The essential idea of this approximation is using the weighted average to vanish the low order leading terms in asymptotic expansions for the truncation errors of the shifted Grünwald formulae. Another class of WSGD approximations can be found in Tian, Zhou and Deng [25]. Initially, the shifted Grünwald formulae were constructed by Meerschaert and Tadjeran [23] and defined by

$$
\begin{aligned}
& \mathscr{A}_{h, p}^{\alpha} u(x):=\frac{1}{h^{\alpha}} \sum_{l=0}^{\infty} g_{l}^{(\alpha)} u(x-(l-p) h)={ }_{-\infty} D_{x}^{\alpha} u(x)+O(h), \\
& \mathscr{A}_{h, r}^{\alpha} u(x):=\frac{1}{h^{\alpha}} \sum_{l=0}^{\infty} g_{l}^{(\alpha)} u(x+(l-r) h)={ }_{x} D_{+\infty}^{\alpha} u(x)+O(h),
\end{aligned}
$$


where $p, r$ are integers and $g_{l}^{(\alpha)}=(-1)^{l}\left(\begin{array}{l}\alpha \\ l\end{array}\right)$ are the coefficients of the power series of the function $(1-z)^{\alpha}$, i.e.,

$$
(1-z)^{\alpha}=\sum_{l=0}^{\infty}(-1)^{l}\left(\begin{array}{l}
\alpha \\
l
\end{array}\right) z^{l}=\sum_{l=0}^{\infty} g_{l}^{(\alpha)} z^{l},
$$

for $|z|<1$, and they can be evaluated recursively

$$
g_{0}^{(\alpha)}=1, \quad g_{l}^{(\alpha)}=\left(1-\frac{\alpha+1}{l}\right) g_{l-1}^{(\alpha)}, \quad l=1,2, \ldots
$$

By weighting the Grünwald approximation formulae with different shifts, Hao, Sun and Cao [14] propose the following WSGD operator:

$$
\begin{aligned}
& { }_{L} \mathscr{D}_{h}^{\alpha} u(x)=\lambda_{1 L} \mathscr{A}_{h, 1}^{\alpha} u(x)+\lambda_{0 L} \mathscr{A}_{h, 0}^{\alpha} u(x)+\lambda_{-1 L^{2}} \mathscr{A}_{h,-1}^{\alpha} u(x), \\
& { }_{R} \mathscr{D}_{h}^{\alpha} u(x)=\lambda_{1 R} \mathscr{A}_{h, 1}^{\alpha} u(x)+\lambda_{0 R} \mathscr{A}_{h, 0}^{\alpha} u(x)+\lambda_{-1 R} \mathscr{A}_{h,-1}^{\alpha} u(x),
\end{aligned}
$$

where

$$
\lambda_{1}=\frac{\alpha^{2}+3 \alpha+2}{12}, \quad \lambda_{0}=\frac{4-\alpha^{2}}{6}, \quad \lambda_{-1}=\frac{\alpha^{2}-3 \alpha+2}{12} .
$$

Lemma 2.1. (See [14].) Suppose that $u \in L^{1}(\mathbb{R})$ and

$$
u \in \mathscr{L}^{2+\alpha}(\mathbb{R}):=\left\{u\left|\int_{-\infty}^{+\infty}(1+|\xi|)^{2+\alpha}\right| \widehat{u}(\xi) \mid d \xi<\infty\right\}
$$

then for a fixed $h$, we have

$$
\begin{aligned}
& { }_{L} \mathscr{D}_{h}^{\alpha} u(x)={ }_{-\infty} D_{x}^{\alpha} u(x)+O\left(h^{2}\right), \\
& { }_{R} \mathscr{D}_{h}^{\alpha} u(x)={ }_{x} D_{+\infty}^{\alpha} u(x)+O\left(h^{2}\right) .
\end{aligned}
$$

Rearranging the WSGD operator gives

$$
\begin{aligned}
& { }_{L} \mathscr{D}_{h}^{\alpha} u(x):=\frac{1}{h^{\alpha}} \sum_{l=0}^{\infty} w_{l}^{(\alpha)} u(x-(l-1) h)={ }_{-\infty} D_{x}^{\alpha} u(x)+O\left(h^{2}\right), \\
& { }_{R} \mathscr{D}_{h}^{\alpha} u(x):=\frac{1}{h^{\alpha}} \sum_{l=0}^{\infty} w_{l}^{(\alpha)} u(x+(l-1) h)={ }_{x} D_{+\infty}^{\alpha} u(x)+O\left(h^{2}\right),
\end{aligned}
$$

where

$$
\left\{\begin{array}{c}
w_{0}^{(\alpha)}=\lambda_{1} g_{0}^{(\alpha)}, \quad w_{1}^{(\alpha)}=\lambda_{1} g_{1}^{(\alpha)}+\lambda_{0} g_{0}^{(\alpha)}, \\
w_{l}^{(\alpha)}=\lambda_{1} g_{l}^{(\alpha)}+\lambda_{0} g_{l-1}^{(\alpha)}+\lambda_{-1} g_{l-2}^{(\alpha)}, \quad l \geqslant 2 .
\end{array}\right.
$$

In addition, the coefficients have the following properties:

$$
\left\{\begin{array}{l}
w_{0}^{(\alpha)} \geqslant 0, \quad w_{1}^{(\alpha)} \leqslant 0, \quad w_{l}^{(\alpha)} \geqslant 0, \quad l \geqslant 3, \\
\sum_{l=0}^{+\infty} w_{l}^{(\alpha)}=0, \quad \sum_{l=0}^{M} w_{l}^{(\alpha)} \leqslant 0, \quad M \geqslant 1, \\
w_{0}^{(\alpha)}+w_{2}^{(\alpha)} \geqslant 0 .
\end{array}\right.
$$


Remark 2.1. For $1<\alpha<2$, it is easy to verify that the inequalities in (2.8) are strictly true, i.e., the sign " $\leqslant$ " and " $\geqslant$ " can be substituted by " <" and " $>$ ".

Using the relation (1.3) and (2.6), the WSGD approximation for the fractional Laplacian can be given by

$$
\begin{aligned}
\Delta_{h}^{\alpha} u(x) & :=\frac{1}{2 \cos \frac{\alpha \pi}{2}}\left({ }_{L} \mathscr{D}_{h}^{\alpha} u(x)+{ }_{R} \mathscr{D}_{h}^{\alpha} u(x)\right) \\
& =\frac{1}{h^{\alpha}} \frac{1}{2 \cos \frac{\alpha \pi}{2}}\left(\sum_{l=0}^{\infty} w_{l}^{(\alpha)} u(x-(l-1) h)+\sum_{l=0}^{\infty} w_{l}^{(\alpha)} u(x+(l-1) h)\right) \\
& =(-\Delta)^{\frac{\alpha}{2}} u(x)+O\left(h^{2}\right) .
\end{aligned}
$$

\subsection{Fractional Sobolev norm}

Now we introduce some fractional Sobolev norms and relevant lemmas.

Let $\mathbb{Z}$ denote the set of all integers and $h \mathbb{Z}$ denote the infinite grid with grid points $x_{j}=j h$ for $j \in \mathbb{Z}$. For any grid functions $u=\left\{u_{j}\right\}, v=\left\{v_{j}\right\}$ on $h \mathbb{Z}$, we define the discrete inner product and the associated $l_{h}^{2}$ norm as

$$
(u, v)_{h}=h \sum_{j \in \mathbb{Z}} u_{j} \bar{v}_{j}, \quad\|u\|_{h}^{2}=(u, u)_{h} .
$$

We also define the discrete $l_{h}^{p}$ norm as

$$
\|u\|_{l_{h}^{p}}^{p}=h \sum_{j \in \mathbb{Z}}\left|u_{j}\right|^{p}, \quad 1 \leqslant p<+\infty
$$

and the discrete $l_{h}^{\infty}$ norm as

$$
\|u\|_{l_{h}^{\infty}}=\sup _{j \in \mathbb{Z}}\left|u_{j}\right|
$$

Define the space $l_{h}^{2}:=\left\{u \mid u=\left\{u_{j}\right\},\|u\|_{h}<+\infty\right\}$. For any $u \in l_{h}^{2}$, the semi-discrete Fourier transform of $u$ is the function $\widehat{u} \in L^{2}[-\pi / h, \pi / h]$ defined by

$$
\widehat{u}(k):=\frac{1}{\sqrt{2 \pi}} h \sum_{j \in \mathbb{Z}} u_{j} e^{-i k x_{j}}
$$

see [35, 36]. Moreover, we have the inversion formula

$$
u_{j}=\frac{1}{\sqrt{2 \pi}} \int_{-\pi / h}^{+\pi / h} \widehat{u}(k) e^{i k x_{j}} \mathrm{~d} k
$$

and Parseval's theorem gives

$$
(u, v)_{h}=\int_{-\pi / h}^{+\pi / h} \widehat{u}(k) \overline{\widehat{v}(k)} \mathrm{d} k .
$$

Given a constant $\sigma \in[0,1]$, we define the fractional Sobolev semi-norm $|\cdot|_{H_{h}^{\sigma}}$ and norm $\|\cdot\|_{H_{h}^{\sigma}}$ as

$$
|u|_{H_{h}^{\sigma}}^{2}=\int_{-\pi / h}^{+\pi / h}|k|^{2 \sigma}|\widehat{u}(k)|^{2} \mathrm{~d} k, \quad \underset{5}{\|u\|_{H_{h}^{\sigma}}^{2}}=\int_{-\pi / h}^{+\pi / h}\left(1+|k|^{2 \sigma}\right)|\widehat{u}(k)|^{2} \mathrm{~d} k .
$$


From (2.10), it is clear that $\|u\|_{H_{h}^{\sigma}}^{2}=\|u\|_{h}^{2}+|u|_{H_{h}^{\sigma}}^{2}$ and $|u|_{H_{h}^{0}}^{2}=\|u\|_{h}^{2}$. Then we introduce the following lemmas.

Lemma 2.2. For $0 \leqslant \sigma_{0} \leqslant \sigma \leqslant 1$, there exists a constant $C \in[1, \sqrt{2}]$ such that

$$
\|u\|_{H_{h}^{\sigma_{0}}} \leqslant C\|u\|_{H_{h}^{\sigma}}^{\frac{\sigma_{0}}{\sigma}}\|u\|_{h}^{1-\frac{\sigma_{0}}{\sigma}} .
$$

Proof. From the definition of $\|u\|_{H_{h}^{\sigma_{0}}}$ and Hölder's inequality, we have

$$
\begin{aligned}
\|u\|_{H_{h}^{\sigma_{0}}}^{2} & =\int_{-\pi / h}^{+\pi / h}\left(1+|k|^{2 \sigma_{0}}\right)|\widehat{u}(k)|^{2} \mathrm{~d} k \\
& =\int_{-\pi / h}^{+\pi / h}\left(\left(1+|k|^{2 \sigma}\right)|\widehat{u}(k)|^{2}\right)^{\frac{\sigma_{0}}{\sigma}}\left(|\widehat{u}(k)|^{2}\right)^{1-\frac{\sigma_{0}}{\sigma}}\left(\frac{1+|k|^{2 \sigma_{0}}}{\left(1+|k|^{2 \sigma}\right)^{\frac{\sigma_{0}}{\sigma}}}\right) \mathrm{d} k \\
& \leqslant C^{2}\left(\int_{-\pi / h}^{+\pi / h}\left(1+|k|^{2 \sigma}\right)|\widehat{u}(k)|^{2} \mathrm{~d} k\right)^{\frac{\sigma_{0}}{\sigma}}\left(\int_{-\pi / h}^{+\pi / h}|\widehat{u}(k)|^{2} \mathrm{~d} k\right)^{1-\frac{\sigma_{0}}{\sigma}} \\
& =C^{2}\left(\|u\|_{H_{h}^{\sigma}}^{\frac{\sigma_{0}}{\sigma}}\|u\|_{h}^{1-\frac{\sigma_{0}}{\sigma}}\right)^{2},
\end{aligned}
$$

where we have used the inequality $\frac{1}{2}\left(1+a^{\mu}\right) \leqslant(1+a)^{\mu} \leqslant\left(1+a^{\mu}\right)$ for $a>0,0 \leqslant \mu \leqslant 1$ to derive the third line of above inequality. Thus the proof is complete.

Lemma 2.3. For any $\frac{p-2}{2 p}<\sigma_{0} \leqslant 1$, there exists a constant $C_{\sigma_{0}}=C\left(\sigma_{0}\right)>0$ independent of $h>0$, such that

$$
\|u\|_{l_{h}^{p}} \leqslant C_{\sigma_{0}}\|u\|_{H_{h}^{\sigma}}^{\frac{\sigma_{0}}{\sigma}}\|u\|_{h}^{1-\frac{\sigma_{0}}{\sigma}}, \quad 2 \leqslant p \leqslant+\infty
$$

for every $\sigma_{0} \leqslant \sigma \leqslant 1$.

Proof. Using the Hausdorff-Young inequality (see Appendix A), for $1 \leqslant q \leqslant 2$ such that $\frac{1}{p}+\frac{1}{q}=1$, we have

$$
\begin{aligned}
\left(h \sum_{j \in \mathbb{Z}}\left|u_{j}\right|^{p}\right)^{\frac{1}{p}} & \leqslant C\left(\int_{-\pi / h}^{+\pi / h}|\widehat{u}(k)|^{q} \mathrm{~d} k\right)^{\frac{1}{q}} \\
& =C\left(\int_{-\pi / h}^{+\pi / h} \frac{1}{\left(1+|k|^{2 \sigma_{0}}\right)^{\frac{q}{2}}}\left(1+|k|^{2 \sigma_{0}}\right)^{\frac{q}{2}}|\widehat{u}(k)|^{q} \mathrm{~d} k\right)^{\frac{1}{q}} .
\end{aligned}
$$

From the Hölder's inequality, it follows that

$$
\begin{aligned}
\left(h \sum_{j \in \mathbb{Z}}\left|u_{j}\right|^{p}\right)^{\frac{1}{p}} & \leqslant C\left(\int_{-\pi / h}^{+\pi / h}\left(1+|k|^{2 \sigma_{0}}\right)|\widehat{u}(k)|^{2} \mathrm{~d} k\right)^{\frac{1}{2}}\left(\int_{-\pi / h}^{+\pi / h} \frac{1}{\left(1+|k|^{2 \sigma_{0}}\right)^{\frac{q}{2-q}}} \mathrm{~d} k\right)^{\frac{2-q}{2 q}} \\
& \leqslant C\|u\|_{H_{h}^{\sigma_{0}}}\left(\int_{-\infty}^{+\infty} \frac{1}{\left(1+|z|^{2 \sigma_{0}}\right)^{\frac{q}{2-q}}} \mathrm{~d} z\right)^{\frac{2-q}{2 q}} .
\end{aligned}
$$


Then for $\frac{p-2}{2 p}<\sigma_{0} \leqslant 1$, we obtain

$$
\|u\|_{l_{h}^{p}} \leqslant \tilde{C}_{\sigma_{0}}\|u\|_{H_{h}^{\sigma_{0}}}
$$

where $\tilde{C}_{\sigma_{0}}=\tilde{C}\left(\sigma_{0}\right)>0$ is independent of $h$. Combining above inequality with (2.12) gives (2.14) and thus completes the proof.

Remark 2.2. Lemma 2.3 is an extension of Lemma 3.2 in [37], where the special case with $p=4$ is considered.

Lemma 2.4. For $1<\alpha \leqslant 2$, let $h(\alpha, \omega)$ be the function defined by

$$
h(\alpha, \omega)=\lambda_{1} \cos \left(\frac{\alpha}{2}(\omega-\pi)-\omega\right)+\lambda_{0} \cos \left(\frac{\alpha}{2}(\omega-\pi)\right)+\lambda_{-1} \cos \left(\frac{\alpha}{2}(\omega-\pi)+\omega\right),
$$

where $\omega \in[0, \pi]$ and $\lambda_{1}, \lambda_{0}, \lambda_{-1}$ are defined in (2.4). Then $h(\alpha, \omega)$ does not decrease with respect to $\omega$.

The proof of above lemma is elementary but quite technical, and hence deferred to the appendix.

Lemma 2.5. For $1<\alpha \leqslant 2$, we have

$$
C_{\alpha}|u|_{H_{h}^{\alpha / 2}}^{2} \leqslant\left(\Delta_{h}^{\alpha} u, u\right)_{h} \leqslant|u|_{H_{h}^{\alpha / 2}}^{2},
$$

where $C_{\alpha}=\frac{2^{\alpha}\left(1-\alpha^{2}\right)}{3 \pi^{\alpha} \cos \frac{\alpha \pi}{2}}>0$.

Proof. From the Parseval's identity (2.10), it follows that

$$
\left(\Delta_{h}^{\alpha} u, u\right)_{h}=\int_{-\pi / h}^{+\pi / h} h^{-\alpha} f(\alpha, k) \widehat{u}(k) \overline{\widehat{u}(k)} \mathrm{d} k
$$

where

$$
f(\alpha, k)=\frac{1}{2 \cos \frac{\alpha \pi}{2}}\left(\sum_{j=0}^{\infty} w_{j}^{(\alpha)} e^{i(j-1) h k}+\sum_{j=0}^{\infty} w_{j}^{(\alpha)} e^{-i(j-1) h k}\right) .
$$

In view of (2.7) and (2.2), we get

$$
\begin{aligned}
f(\alpha, k)= & \frac{1}{2 \cos \frac{\alpha \pi}{2}}\left[\lambda_{1} e^{-i h k} \sum_{j=0}^{\infty} g_{j}^{(\alpha)} e^{i j h k}+\lambda_{0} \sum_{j=0}^{\infty} g_{j}^{(\alpha)} e^{i j h k}+\lambda_{-1} e^{i h k} \sum_{j=0}^{\infty} g_{j}^{(\alpha)} e^{i j h k}\right. \\
& \left.+\lambda_{1} e^{i h k} \sum_{j=0}^{\infty} g_{j}^{(\alpha)} e^{-i j h k}+\lambda_{0} \sum_{j=0}^{\infty} g_{j}^{(\alpha)} e^{-i j h k}+\lambda_{-1} e^{-i h k} \sum_{j=0}^{\infty} g_{j}^{(\alpha)} e^{-i j h k}\right] \\
= & \frac{1}{2 \cos \frac{\alpha \pi}{2}}\left[\lambda_{1}\left(e^{-i h k}\left(1-e^{i h k}\right)^{\alpha}+e^{i k}\left(1-e^{-i h k}\right)^{\alpha}\right)+\lambda_{0}\left(\left(1-e^{i h k}\right)^{\alpha}+\left(1-e^{-i h k}\right)^{\alpha}\right)\right. \\
& \left.+\lambda_{-1}\left(e^{i h k}\left(1-e^{i h k}\right)^{\alpha}+e^{-i k}\left(1-e^{-i h k}\right)^{\alpha}\right)\right] .
\end{aligned}
$$


Clearly, $f(\alpha, k)$ is a real-valued even function, and it is therefore sufficient to consider its principle value for $k \in[0, \pi / h]$. Invoking the relation $e^{i \theta}-e^{i \phi}=2 i \sin \left(\frac{\theta-\phi}{2}\right) e^{\frac{i(\theta+\phi)}{2}}$, we have

$$
\begin{aligned}
f(\alpha, k) & =\frac{\left(2 \sin \frac{h k}{2}\right)^{\alpha}}{\cos \frac{\alpha \pi}{2}}\left(\lambda_{1} \cos \left(\frac{\alpha}{2}(h k-\pi)-h k\right)+\lambda_{0} \cos \left(\frac{\alpha}{2}(h k-\pi)\right)+\lambda_{-1} \cos \left(\frac{\alpha}{2}(h k-\pi)+h k\right)\right) \\
& =\frac{\left(2 \sin \frac{h k}{2}\right)^{\alpha}}{\cos \frac{\alpha \pi}{2}} h(\alpha, h k),
\end{aligned}
$$

where $h(\alpha, h k)$ is defined as in 2.17) with $\omega=h k \in[0, \pi]$. From Lemma 2.4 it follows that

$$
\cos \left(\frac{\alpha \pi}{2}\right)=h(\alpha, 0) \leqslant h(\alpha, h k) \leqslant h(\alpha, \pi)=\frac{1-\alpha^{2}}{3} .
$$

Hence, combining above inequality with the fact that for $k \in[0, \pi / h], h k / \pi \leqslant \sin (h k / 2) \leqslant h k / 2$, we obtain

$$
C_{\alpha}|h k|^{\alpha} \leqslant f(\alpha, k) \leqslant|h k|^{\alpha} .
$$

This together with (2.19) implies $(2.18)$ and thus completes the proof.

Remark 2.3. This idea can be used to analyze some other popular second order schemes, including the WSGD methods proposed in [25] and the fractional centered difference method [31, 32], and similar results can be derived. Unfortunately, it seems difficult to extend directly this study to fourth order schemes, such as the weighted and shifted Lubich difference method [27, 28], because the involved function $f(\alpha, k)$ is much more complicated.

\section{Finite difference scheme}

In practical computation, the whole space problem is usually truncated onto a finite interval $\Omega=(a, b)$ subject to the homogeneous boundary condition ( $a$ and $b$ are usually chosen sufficient large such that the truncation error is negligible). Thus the FGLE (1.1)-(1.2) is truncated on the interval $\Omega=(a, b)$ as

$$
\begin{aligned}
& u_{t}+(v+i \eta)(-\Delta)^{\frac{\alpha}{2}} u+(\kappa+i \zeta)|u|^{2} u-\gamma u=0, \quad x \in \Omega, t \in(0, T] \\
& u(x, 0)=u_{0}(x), \quad x \in \mathbb{R}, \\
& u(x, t)=0, \quad x \in \mathbb{R} \backslash \Omega, t \in[0, T] .
\end{aligned}
$$

The boundary condition (3.3) is referred to as the nonlocal volume constraint (or the extended Dirichlet boundary) and the corresponding problem (3.1)-(3.3) as the volume constraint problem (see [38, 39] for more details). It is noted that, under this boundary, the fractional derivative has reduced to $-(-\Delta)^{\frac{\alpha}{2}} u(x, t)=-\frac{1}{2 \cos \frac{\alpha \pi}{2}}\left[{ }_{a} D_{x}^{\alpha} u(x, t)+{ }_{x} D_{b}^{\alpha} u(x, t)\right]$.

Let $h=\frac{b-a}{M}$ with a positive integer $M$ and define $x_{j}=a+j h, 0 \leqslant j \leqslant M$. Owing to the above boundary constraint (3.3), if $u \in \mathscr{L}^{2+\alpha}(\mathbb{R})$ (see Remark 2.5 in [14]), the WSGD operator (2.6) 
can be simplified as

$$
\begin{aligned}
& { }_{L} \mathscr{D}_{h}^{\alpha} u\left(x_{j}\right):=\frac{1}{h^{\alpha}} \sum_{l=0}^{j+1} w_{l}^{(\alpha)} u\left(x_{j-l+1}\right)={ }_{a} D_{x}^{\alpha} u\left(x_{j}\right)+O\left(h^{2}\right), \\
& { }_{R} \mathscr{D}_{h}^{\alpha} u\left(x_{j}\right):=\frac{1}{h^{\alpha}} \sum_{l=0}^{M-j+1} w_{l}^{(\alpha)} u\left(x_{j+l-1}\right)={ }_{x} D_{b}^{\alpha} u\left(x_{j}\right)+O\left(h^{2}\right),
\end{aligned}
$$

and WSGD approximation (2.9) for the fractional Laplacian as

$$
\begin{aligned}
\Delta_{h}^{\alpha} u\left(x_{j}\right) & =\frac{1}{h^{\alpha}} \frac{1}{2 \cos \frac{\alpha \pi}{2}}\left(\sum_{l=0}^{j+1} w_{l}^{(\alpha)} u\left(x_{j-l+1}\right)+\sum_{l=0}^{M-j+1} w_{l}^{(\alpha)} u\left(x_{j+l-1}\right)\right) \\
& =(-\Delta)^{\frac{\alpha}{2}} u\left(x_{j}\right)+O\left(h^{2}\right) .
\end{aligned}
$$

Chosen the time step $\tau:=\frac{T}{N}$ with a positive integer $N$, define a partition of $[0, T] \times[a, b]$ by $\Omega_{\tau} \times \Omega_{h}$ with the grid $\Omega_{\tau}=\left\{t_{n} \mid t_{n}=n \tau, 0 \leqslant n \leqslant N\right\}$ and $\Omega_{h}=\left\{x_{j} \mid x_{j}=a+j h, 0 \leqslant j \leqslant M\right\}$.

Given a grid function $v=\left\{v_{j}^{n} \mid\left(x_{j}, t_{n}\right) \in \Omega_{\tau} \times \Omega_{h}\right\}$, denote

$$
\delta_{t} v_{j}^{n+\frac{1}{2}}=\frac{v_{j}^{n+1}-v_{j}^{n}}{\tau}, \quad v_{j}^{n+\frac{1}{2}}=\frac{v_{j}^{n+1}+v_{j}^{n}}{2} .
$$

Denote the index set $\mathscr{T}_{M}=\{j \mid j=1,2, \ldots, M-1\}$ and the grid function space $\mathscr{V}_{h}=\{v \mid v=$ $\left.\left(v_{1}, v_{2}, \ldots, v_{M-1}\right)\right\}$.

Under the boundary constraint (3.3), the inner product $(\cdot, \cdot)_{h}$ and norms $\|\cdot\|_{h},\|\cdot\|_{l_{h}^{p}},\|\cdot\|_{l_{h}^{\infty}}$ previously defined in the unbounded interval carry over to the finite interval by regarding that $u_{j}=0$ for $j \leqslant 0$ and $j \geqslant M$. Hence in these notations, we just restrict the index $j$ from 1 to $M-1$ and continue to use these notations without confusion for convenience. Based on these considerations, the inequalities introduced in above section still hold in the finite interval.

With these premises, we now propose a difference scheme for the FGLE (3.1). Let $u_{j}^{n}$ be the numerical approximation of $u\left(x_{j}, t_{n}\right)$. Applying the implicit midpoint method in time and the WSGD approximation for the fractional Laplacian, the difference scheme reads

$$
\begin{aligned}
& \delta_{t} u_{j}^{n+\frac{1}{2}}+(v+i \eta) \Delta_{h}^{\alpha} u_{j}^{n+\frac{1}{2}}+(\kappa+i \zeta)\left|u_{j}^{n+\frac{1}{2}}\right|^{2} u_{j}^{n+\frac{1}{2}}-\gamma u_{j}^{n+\frac{1}{2}}=0, \\
& j \in \mathscr{T}_{M}, 0 \leqslant n \leqslant N-1, \\
& u_{j}^{0}=u_{0}\left(x_{j}\right), \quad j \in \mathbb{Z}, \\
& u_{j}^{n}=0, \quad j \in \mathbb{Z} \backslash \mathscr{T}_{M}, \quad 0 \leqslant n \leqslant N .
\end{aligned}
$$

\section{Theoretical analysis}

In this section, we study theoretical properties of the scheme (3.7)-(3.9), including the a priori estimate, solvability and convergence. 


\subsection{A priori bound}

For showing the a priori bound of the solution to the scheme (3.7)-(3.9), we first introduce some notations and lemmas.

Denote matrix

$$
\mathbf{W}=\left(\begin{array}{ccccc}
w_{1}^{(\alpha)} & w_{0}^{(\alpha)} & & & \\
w_{2}^{(\alpha)} & w_{1}^{(\alpha)} & w_{0}^{(\alpha)} & & \\
\vdots & w_{2}^{(\alpha)} & w_{1}^{(\alpha)} & \ddots & \\
w_{M-2}^{(\alpha)} & \vdots & \ddots & \ddots & w_{0}^{(\alpha)} \\
w_{M-1}^{(\alpha)} & w_{M-2}^{(\alpha)} & \cdots & w_{2}^{(\alpha)} & w_{1}^{(\alpha)}
\end{array}\right) \in \mathbb{R}^{(M-1) \times(M-1)}
$$

and matrix $\mathbf{C}=\frac{1}{2 \cos \frac{\alpha \pi}{2}}\left(\mathbf{W}+\mathbf{W}^{T}\right) \in \mathbb{R}^{(M-1) \times(M-1)}$. Then for $u=\left(u_{1}, u_{2}, \ldots, u_{M-1}\right)^{T}$, we can rewrite $\Delta_{h}^{\alpha} u=\frac{1}{h^{\alpha}} \mathbf{C} u$. In addition, we have the following lemmas.

Lemma 4.1. Matrix $\mathbf{C}$ is a real-valued symmetry positive definite matrix.

Proof. It is obviously seen that $\mathbf{C}$ is a real-valued symmetry matrix. The positive definiteness, for $1<\alpha<2$, can be obtained by invoking the property of coefficients in (2.8) and the Geršgorin disc theorem [40]. For $\alpha=2$, matrix $\mathbf{C}$ reduces to the classical Laplacian matrix associated with the second order centered difference and thus, the positive definiteness is obtained.

Lemma 4.2. For any two grid functions $u, v \in \mathscr{V}_{h}$, there exists a linear operator $\Lambda^{\alpha}$ such that

$$
\left(\Delta_{h}^{\alpha} u, v\right)_{h}=\left(\Lambda^{\alpha} u, \Lambda^{\alpha} v\right)_{h} .
$$

Proof. The proof is similar to that in [41] (see Lemma 3.1 in [41]) where the fractional centered difference is adopted. The linear operator $\Lambda^{\alpha}$ is defined by $\Lambda^{\alpha} u=h^{-\frac{\alpha}{2}} \mathbf{L} u$ where matrix $\mathbf{L}$ satisfying $\mathbf{C}=\mathbf{L}^{T} \mathbf{L}$ is the Cholesky factor.

Based on the previous lemma, we can establish the following boundedness estimate.

Theorem 4.1. The difference solution of scheme (3.7)-(3.9) is bounded in the following sense

$$
\left\|u^{n}\right\|_{h} \leqslant C_{M}, \quad 0 \leqslant n \leqslant N
$$

Proof. Computing the discrete inner product of (3.7) with $u^{n+\frac{1}{2}}$, then taking the real part of the resulting equation, we obtain

$$
\frac{\left\|u^{n+1}\right\|_{h}^{2}-\left\|u^{n}\right\|_{h}^{2}}{2 \tau}+v\left\|\Lambda^{\alpha} u^{n+\frac{1}{2}}\right\|_{h}^{2}+\kappa\left\|u^{n+\frac{1}{2}}\right\|_{l_{h}^{4}}^{4}=\gamma\left\|u^{n+\frac{1}{2}}\right\|_{h}^{2}, \quad 0 \leqslant n \leqslant N-1,
$$

where we have used the relation (4.1).

If $\gamma \leqslant 0$, from the above inequality we get

$$
\left\|u^{n+1}\right\|_{h}^{2} \leqslant\left\|u^{n}\right\|_{h}^{2} \leqslant \cdots \leqslant\left\|u_{10}^{0}\right\|_{h}^{2}, \quad 0 \leqslant n \leqslant N-1 .
$$


If $\gamma>0$, it follows from (4.3) that

$$
\left\|u^{n+1}\right\|_{h}^{2}-\left\|u^{n}\right\|_{h}^{2} \leqslant 2 \tau \gamma\left\|u^{n+\frac{1}{2}}\right\|_{h}^{2} \leqslant \tau \gamma\left(\left\|u^{n+1}\right\|_{h}^{2}+\left\|u^{n}\right\|_{h}^{2}\right), \quad 0 \leqslant n \leqslant N-1 .
$$

Let $\tau \leqslant \frac{1}{2 \gamma}$. We have

$$
\left\|u^{n+1}\right\|_{h}^{2} \leqslant(1+4 \gamma \tau)\left\|u^{n}\right\|_{h}^{2}, \quad 0 \leqslant n \leqslant N-1,
$$

which immediately implies

$$
\left\|u^{n}\right\|_{h}^{2} \leqslant \exp (4 \gamma T)\left\|u^{0}\right\|_{h}^{2}, \quad 0 \leqslant n \leqslant N .
$$

Thus the proof is complete.

Remark 4.1. Theorem 4.1 implies that the difference solution of scheme (3.7)-(3.9) is bounded for a long time if $\gamma \leqslant 0$, while is bounded for a given $T$ if $\gamma>0$.

\subsection{Solvability}

The existence of the solution is shown by virtue of the Brouwder fixed point theorem.

Lemma 4.3. (Brouwder fixed point theorem [42]) Let $(\mathscr{H},\langle\cdot, \cdot\rangle)$ be a finite dimensional inner product space, $\|\cdot\|$ be the associated norm, and $f: \mathscr{H} \rightarrow \mathscr{H}$ be continuous. Assume, moreover, that

$$
\exists \rho>0, \forall z \in \mathscr{H},\|z\|=\rho, \operatorname{Re}\langle g(z), z\rangle \geqslant 0 .
$$

Then, there exists a $z^{*} \in \mathscr{H}$ such that $g\left(z^{*}\right)=0$ and $\left\|z^{*}\right\| \leqslant \rho$.

Theorem 4.2. The solution of difference scheme (3.7)-(3.9) exists.

Proof. The proof proceeds in an inductive way. Obviously, $u^{0}$ has been determined uniquely from (3.8) and (3.9). For given $u^{n}(0 \leqslant n \leqslant N-1)$, it remains to prove that there exists $u^{n+1}$ satisfying the scheme. To this end, for fixed $n$, rewrite 3.7 in the form

$$
u_{j}^{n+\frac{1}{2}}=u_{j}^{n}-\frac{\tau}{2}\left[(v+i \eta) \Delta_{h}^{\alpha} u_{j}^{n+\frac{1}{2}}+(\kappa+i \zeta)\left|u_{j}^{n+\frac{1}{2}}\right|^{2} u_{j}^{n+\frac{1}{2}}-\gamma u_{j}^{n+\frac{1}{2}}\right], \quad j \in \mathscr{T}_{M} .
$$

Consider a mapping $\mathscr{F}: \mathscr{V}_{h} \rightarrow \mathscr{V}_{h}$ defined by

$$
(\mathscr{F}(v))_{j}=v_{j}-u_{j}^{n}+\frac{\tau}{2}\left[(v+i \eta) \Delta_{h}^{\alpha} v_{j}+(\kappa+i \zeta)\left|v_{j}\right|^{2} v_{j}-\gamma v_{j}\right], \quad j \in \mathscr{T}_{M},
$$

which is obviously continuous. Computing the discrete inner product of (4.5) with $v$ gives

$$
(\mathscr{F}(v), v)_{h}=\|v\|_{h}^{2}-\left(u^{n}, v\right)_{h}+\frac{\tau}{2}\left[(v+i \eta)\left\|\Lambda^{\alpha} v\right\|_{h}^{2}+(\kappa+i \zeta)\|v\|_{l_{h}^{4}}^{4}-\gamma\|v\|_{h}^{2}\right],
$$

where (4.1) was used. Then, taking the real part, we obtain

$$
\begin{aligned}
& \operatorname{Re}(\mathscr{F}(v), v)_{h}=\left(1-\frac{\gamma \tau}{2}\right)\|v\|_{h}^{2}-\operatorname{Re}\left(u^{n}, v\right)+\frac{v \tau}{2}\left\|\Lambda^{\alpha} v\right\|_{h}^{2}+\frac{\kappa \tau}{2}\|v\|_{l_{h}^{4}}^{4} \\
& \geqslant\left(1-\frac{\gamma \tau}{2}\right)\|v\|_{h}^{2}-\left\|u^{n}\right\|_{h}\|v\|_{h} \\
&=\|v\|_{h}\left(\left(1-\frac{\gamma \tau}{2}\right)\|v\|_{h}-\left\|u^{n}\right\|_{h}\right) . \\
& 11
\end{aligned}
$$


Hence, taking $\gamma \tau \leqslant 1$ and $\|v\|_{h}=2\left\|u^{n}\right\|_{h}$, there exists $\operatorname{Re}(\mathscr{F}(v), v)_{h} \geqslant 0$. Then from Lemma 4.3. we obtain the existence of $u^{n+\frac{1}{2}}$ and thus, the existence of $u^{n+1}$ by noting that $u^{n+1}=2 u^{n+\frac{1}{2}}-u^{n}$.

For a technical reason, the uniqueness of the solution will be shown in the subsection 4.4.

\subsection{Convergence}

Before establishing the convergence, we first analyze the local truncation error of scheme (3.7)(3.9). For notational convenience we denote grid functions $U_{j}^{n}:=u\left(x_{j}, t_{n}\right)$. Define the truncation error as

$$
\begin{array}{r}
R_{j}^{n+\frac{1}{2}}:=\delta_{t} U_{j}^{n+\frac{1}{2}}+(v+i \eta) \Delta_{h}^{\alpha} U_{j}^{n+\frac{1}{2}}+(\kappa+i \zeta)\left|U_{j}^{n+\frac{1}{2}}\right|^{2} U_{j}^{n+\frac{1}{2}}-\gamma U_{j}^{n+\frac{1}{2}}, \\
j \in \mathscr{T}_{M}, \quad 0 \leqslant n \leqslant N-1 .
\end{array}
$$

Then from (3.5) and Taylor's expansion, we can obtain the following local truncation error estimate.

Lemma 4.4. Suppose that the problem (3.1)-(3.3) has a smooth solution. Then we have

$$
\left|R_{j}^{n+\frac{1}{2}}\right| \leqslant C_{R}\left(\tau^{2}+h^{2}\right), \quad j \in \mathscr{T}_{M}, 0 \leqslant n \leqslant N-1 .
$$

Proof. Applying the Taylor's expansion of the solution at $\left(x_{j}, t_{n+\frac{1}{2}}\right)$ yields

$$
\begin{aligned}
& \frac{u\left(x_{j}, t_{n+1}\right)-u\left(x_{j}, t_{n}\right)}{\tau} \\
& =\partial_{t} u\left(x_{j}, t_{n+\frac{1}{2}}\right)+\frac{\tau^{2}}{16} \int_{0}^{1}\left[\frac{\partial^{3} u}{\partial t^{3}}\left(x_{j}, t_{n+\frac{1}{2}}+\frac{s}{2} \tau\right)+\frac{\partial^{3} u}{\partial t^{3}}\left(x_{j}, t_{n+\frac{1}{2}}-\frac{s}{2} \tau\right)\right](1-s)^{2} \mathrm{~d} s,
\end{aligned}
$$

and

$$
\begin{aligned}
& \frac{u\left(x_{j}, t_{n+1}\right)+u\left(x_{j}, t_{n}\right)}{2} \\
& =u\left(x_{j}, t_{n+\frac{1}{2}}\right)+\frac{\tau^{2}}{8} \int_{0}^{1}\left[\frac{\partial^{2} u}{\partial t^{2}}\left(x_{j}, t_{n+\frac{1}{2}}+\frac{s}{2} \tau\right)+\frac{\partial^{2} u}{\partial t^{2}}\left(x_{j}, t_{n+\frac{1}{2}}-\frac{s}{2} \tau\right)\right](1-s) \mathrm{d} s .
\end{aligned}
$$

Furthermore, noticing the error estimate (3.5), we have

$$
\begin{aligned}
& \Delta_{h}^{\alpha}\left(\frac{u\left(x_{j}, t_{n+1}\right)+u\left(x_{j}, t_{n}\right)}{2}\right)-(-\Delta)^{\frac{\alpha}{2}} u\left(x_{j}, t_{n+\frac{1}{2}}\right) \\
& =\frac{\tau^{2}}{8} \int_{0}^{1}(-\Delta)^{\frac{\alpha}{2}}\left[\frac{\partial^{2} u}{\partial t^{2}}\left(x_{j}, t_{n+\frac{1}{2}}+\frac{s}{2} \tau\right)+\frac{\partial^{2} u}{\partial t^{2}}\left(x_{j}, t_{n+\frac{1}{2}}-\frac{s}{2} \tau\right)\right](1-s) \mathrm{d} s+O\left(h^{2}\right),
\end{aligned}
$$

Substituting (4.10)-(4.12) into (4.8) gives (4.9) immediately and thus, completes the proof.

From above lemma, it follows that

$$
\left\|R^{n+\frac{1}{2}}\right\|_{h}^{2} \leqslant(b-a)\left(C_{R}\left(\tau_{12}^{2}+h^{2}\right)\right)^{2}, \quad 0 \leqslant n \leqslant N-1 .
$$


Define the error function $e^{n} \in \mathscr{V}_{h}$ as

$$
e_{j}^{n}=U_{j}^{n}-u_{j}^{n}, \quad j \in \mathscr{T}_{M}, \quad 0 \leqslant n \leqslant N .
$$

Then we get the following convergence result.

Theorem 4.3. Suppose that the problem (3.1)-(3.3) has a smooth solution. Then there exists $\tau_{0}>0$ sufficiently small such that, when $0<\tau \leqslant \tau_{0}$, we have

$$
\left\|e^{n}\right\|_{h} \leqslant C\left(\tau^{2}+h^{2}\right), \quad 0 \leqslant n \leqslant N
$$

where $C$ denotes a positive constant independent of $\tau$ and $h$.

Proof. Subtracting (3.7) from (4.8) gives the following error equation

$$
\begin{aligned}
\delta_{t} e_{j}^{n+\frac{1}{2}}+(v+i \eta) \Delta_{h}^{\alpha} e_{j}^{n+\frac{1}{2}}+(\kappa+i \zeta)\left|u_{j}^{n+\frac{1}{2}}\right|^{2} e_{j}^{n+\frac{1}{2}}+(\kappa+i \zeta) G_{j}^{n+\frac{1}{2}} U_{j}^{n+\frac{1}{2}}-\gamma e_{j}^{n+\frac{1}{2}} & =R_{j}^{n+\frac{1}{2}}, \\
j \in \mathscr{T}_{M}, \quad 0 & \leqslant n \leqslant N-1,
\end{aligned}
$$

where

$$
G_{j}^{n+\frac{1}{2}}=\left|U_{j}^{n+\frac{1}{2}}\right|^{2}-\left|u_{j}^{n+\frac{1}{2}}\right|^{2} .
$$

Computing the discrete inner product of (4.15) with $e^{n+\frac{1}{2}}$, then taking the real part of the resulting equation, we obtain

$$
\begin{aligned}
& \frac{\left\|e^{n+1}\right\|_{h}^{2}-\left\|e^{n}\right\|_{h}^{2}}{2 \tau}+v\left\|\Lambda^{\alpha} e^{n+\frac{1}{2}}\right\|_{h}^{2}+\kappa h \sum_{j=1}^{M-1}\left|u_{j}^{n+\frac{1}{2}}\right|^{2}\left|e_{j}^{n+\frac{1}{2}}\right|^{2}-\gamma\left\|e^{n+\frac{1}{2}}\right\|_{h}^{2} \\
& +\operatorname{Re}\left((\kappa+i \zeta) h \sum_{j=1}^{M-1} G_{j}^{n+\frac{1}{2}} U_{j}^{n+\frac{1}{2}} \overline{e_{j}^{n+\frac{1}{2}}}\right)=\operatorname{Re}\left(R^{n+\frac{1}{2}}, e^{n+\frac{1}{2}}\right)_{h}, \quad 0 \leqslant n \leqslant N-1 .
\end{aligned}
$$

We first estimate the last term on the left-hand side of (4.16). In view of the smoothness assumption of the exact solution, denote $C_{u}=\sup _{0 \leqslant t \leqslant T, a \leqslant x \leqslant b}|u(x, t)|$. Noting that

$$
\left|u_{j}^{n}\right| \leqslant\left|U_{j}^{n}\right|+\left|e_{j}^{n}\right| \leqslant C_{u}+\left|e_{j}^{n}\right|, \quad 0 \leqslant n \leqslant N,
$$

we have for $0 \leqslant n \leqslant N-1$,

$$
\begin{aligned}
\left|G_{j}^{n+\frac{1}{2}} U_{j}^{n+\frac{1}{2}}\right| & =\left|\left(\left|U_{j}^{n+\frac{1}{2}}\right|^{2}-\left|u_{j}^{n+\frac{1}{2}}\right|^{2}\right) U_{j}^{n+\frac{1}{2}}\right| \\
& \leqslant C_{u}\left|U_{j}^{n+\frac{1}{2}}-u_{j}^{n+\frac{1}{2}}\right|\left(\left|U_{j}^{n+\frac{1}{2}}\right|+\left|u_{j}^{n+\frac{1}{2}}\right|\right) \\
& \leqslant C_{u}\left|e_{j}^{n+\frac{1}{2}}\right|\left(2 C_{u}+\left|e_{j}^{n+\frac{1}{2}}\right|\right) \\
& \leqslant 2 C_{u}^{2}\left|e_{j}^{n+\frac{1}{2}}\right|+C_{u}\left|e_{j}^{n+\frac{1}{2}}\right|^{2} .
\end{aligned}
$$


Then we obtain

$$
\begin{aligned}
& \left|\operatorname{Re}\left((\kappa+i \zeta) h \sum_{j=1}^{M-1} G_{j}^{n+\frac{1}{2}} U_{j}^{n+\frac{1}{2}} \overline{e_{j}^{n+\frac{1}{2}}}\right)\right| \\
& \leqslant \sqrt{\kappa^{2}+\zeta^{2}} h \sum_{j=1}^{M-1}\left(2 C_{u}^{2}\left|e_{j}^{n+\frac{1}{2}}\right|+C_{u}\left|e_{j}^{n+\frac{1}{2}}\right|^{2}\right)\left|e_{j}^{n+\frac{1}{2}}\right| \\
& =\sqrt{\kappa^{2}+\zeta^{2}}\left(2 C_{u}^{2}\left\|e^{n+\frac{1}{2}}\right\|_{h}^{2}+C_{u}\left\|e^{n+\frac{1}{2}}\right\|_{l_{h}^{3}}^{3}\right), \quad 0 \leqslant n \leqslant N-1 .
\end{aligned}
$$

For the term on the right-hand side of (4.16), using the Cauchy-Swcharz inequality gives

$$
\operatorname{Re}\left(R^{n+\frac{1}{2}}, e^{n+\frac{1}{2}}\right)_{h} \leqslant \frac{1}{2}\left(\left\|R^{n+\frac{1}{2}}\right\|_{h}^{2}+\left\|e^{n+\frac{1}{2}}\right\|_{h}^{2}\right), \quad 0 \leqslant n \leqslant N-1 .
$$

By substituting (4.17) and (4.18) into (4.16), we get for $0 \leqslant n \leqslant N-1$,

$$
\begin{aligned}
& \frac{\left\|e^{n+1}\right\|_{h}^{2}-\left\|e^{n}\right\|_{h}^{2}}{2 \tau}+v\left\|\Lambda^{\alpha} e^{n+\frac{1}{2}}\right\|_{h}^{2} \\
& \leqslant \sqrt{\kappa^{2}+\zeta^{2}}\left(2 C_{u}^{2}\left\|e^{n+\frac{1}{2}}\right\|_{h}^{2}+C_{u}\left\|e^{n+\frac{1}{2}}\right\|_{l_{h}^{3}}^{3}\right)+|\gamma|\left\|e^{n+\frac{1}{2}}\right\|_{h}^{2}+\frac{1}{2}\left(\left\|R^{n+\frac{1}{2}}\right\|_{h}^{2}+\left\|e^{n+\frac{1}{2}}\right\|_{h}^{2}\right) \\
& =\left(2 \sqrt{\kappa^{2}+\zeta^{2}} C_{u}^{2}+|\gamma|+\frac{1}{2}\right)\left\|e^{n+\frac{1}{2}}\right\|_{h}^{2}+\sqrt{\kappa^{2}+\zeta^{2}} C_{u}\left\|e^{n+\frac{1}{2}}\right\|_{l_{h}^{3}}^{3}+\frac{1}{2}\left\|R^{n+\frac{1}{2}}\right\|_{h}^{2} .
\end{aligned}
$$

Furthermore, Theorem 4.1 implies that

$$
\left\|e^{n+\frac{1}{2}}\right\|_{h} \leqslant\left\|U^{n+\frac{1}{2}}\right\|_{h}+\left\|u^{n+\frac{1}{2}}\right\|_{h} \leqslant \sqrt{b-a} C_{u}+C_{M}, \quad 0 \leqslant n \leqslant N-1 .
$$

Hence, in view of (2.14) with $p=3$ and $\sigma_{0}=\frac{\alpha}{6}$, we have

$$
\begin{aligned}
\left\|e^{n+\frac{1}{2}}\right\|_{l_{h}^{3}}^{3} & \leqslant C_{\sigma_{0}}\left\|e^{n+\frac{1}{2}}\right\|_{h}^{2}\left\|e^{n+\frac{1}{2}}\right\|_{H_{h}^{\alpha / 2}} \\
& \leqslant C_{\sigma_{0}}\left(\varepsilon\left|e^{n+\frac{1}{2}}\right|_{H_{h}^{\alpha / 2}}^{2}+\varepsilon\left\|e^{n+\frac{1}{2}}\right\|_{h}^{2}+\frac{1}{4 \varepsilon}\left\|e^{n+\frac{1}{2}}\right\|_{h}^{4}\right) \\
& \leqslant C_{\sigma_{0}}\left(\frac{\varepsilon}{C_{\alpha}}\left\|\Lambda^{\alpha} e^{n+\frac{1}{2}}\right\|_{h}^{2}+\left(\varepsilon+\frac{\left(\sqrt{b-a} C_{u}+C_{M}\right)^{2}}{4 \varepsilon}\right)\left\|e^{n+\frac{1}{2}}\right\|_{h}^{2}\right),
\end{aligned}
$$

where we have used (2.18) and (4.1) for the last inequality. Taking $\varepsilon=\frac{C_{\alpha} v}{\sqrt{\kappa^{2}+\zeta^{2}} C_{u} C_{\sigma_{0}}}$ and plugging the above inequality into (4.19), we obtain

$$
\frac{\left\|e^{n+1}\right\|_{h}^{2}-\left\|e^{n}\right\|_{h}^{2}}{2 \tau}+v\left\|\Lambda^{\alpha} e^{n+\frac{1}{2}}\right\|_{h}^{2} \leqslant v\left\|\Lambda^{\alpha} e^{n+\frac{1}{2}}\right\|_{h}^{2}+C_{1}\left\|e^{n+\frac{1}{2}}\right\|_{h}^{2}+\frac{1}{2}\left\|R^{n+\frac{1}{2}}\right\|_{h}^{2}, \quad 0 \leqslant n \leqslant N-1,
$$

namely,

$$
\left\|e^{n+1}\right\|_{h}^{2}-\left\|e^{n}\right\|_{h}^{2} \leqslant \tau C_{1}\left(\left\|e^{n+1}\right\|_{h}^{2}+\left\|e_{14}^{n}\right\|_{h}^{2}\right)+\tau\left\|R^{n+\frac{1}{2}}\right\|_{h}^{2}, \quad 0 \leqslant n \leqslant N-1
$$


where $C_{1}=2 \sqrt{\kappa^{2}+\zeta^{2}} C_{u}^{2}+|\gamma|+\frac{1}{2}+C_{\alpha} v+\frac{\left(\kappa^{2}+\zeta^{2}\right) C_{u}^{2} C_{\sigma_{0}}^{2}\left(\sqrt{b-a} C_{u}+C_{M}\right)^{2}}{4 C_{\alpha} v}$.

If $\tau \leqslant \frac{1}{2 C_{1}}$, we have

$$
\left\|e^{n+1}\right\|_{h}^{2} \leqslant\left(1+4 C_{1} \tau\right)\left\|e^{n}\right\|_{h}^{2}+2 \tau\left\|R^{n+\frac{1}{2}}\right\|_{h}^{2}, \quad 0 \leqslant n \leqslant N-1 .
$$

This together with (4.13) and the discrete Gronwall inequality gives

$$
\left\|e^{n}\right\|_{h}^{2} \leqslant \exp \left(4 C_{1} T\right) \frac{(b-a) C_{R}^{2}}{2 C_{1}}\left(\tau^{2}+h^{2}\right)^{2}, \quad 0 \leqslant n \leqslant N,
$$

which implies (4.14) with $C=\exp \left(2 C_{1} T\right) C_{R} \sqrt{\frac{b-a}{2 C_{1}}}$. Thus, the proof is complete.

\subsection{Uniqueness}

Now we are in a position to show the uniqueness of the solution to the scheme (3.7)-3.9).

From Theorem 4.3, using the inverse inequality $\|\cdot\|_{l_{h}^{\infty}}^{2} \leqslant h^{-1}\|\cdot\|_{h}^{2}$, we first show the uniform boundedness of the difference solution. In fact, assume $\tau \leqslant C_{2} h$, then for $0<h \leqslant\left(\frac{1}{C\left(1+C_{2}^{2}\right)}\right)^{\frac{2}{3}}$, we obtain

$$
\left\|u^{n}\right\|_{l_{h}^{\infty}} \leqslant\left\|U^{n}\right\|_{l_{h}^{\infty}}+\left\|e^{n}\right\|_{l_{h}^{\infty}} \leqslant C_{u}+C h^{-\frac{1}{2}}\left(\tau^{2}+h^{2}\right) \leqslant 1+C_{u}, \quad 0 \leqslant n \leqslant N .
$$

Using this inequality, we have the following results.

Theorem 4.4. Assume $\tau \leqslant C h$, then there exist $\tau_{0}>0$ and $h_{0}>0$ sufficiently small such that, when $0<\tau \leqslant \tau_{0}$ and $0<h \leqslant h_{0}$, the solution of difference scheme (3.7)-(3.9) is unique.

Proof. Suppose there exist two solutions $u^{(1)}, u^{(2)} \in \mathscr{V}_{h}$ to the scheme (3.7)-(3.9). Then from (4.25), we have

$$
\left\|u^{n}\right\|_{l_{h}^{\infty}} \leqslant 1+C_{u}, \quad\left\|u^{(1)}\right\|_{l_{h}^{\infty}} \leqslant 1+C_{u}, \quad\left\|u^{(2)}\right\|_{l_{h}^{\infty}} \leqslant 1+C_{u}, \quad 0 \leqslant n \leqslant N-1 .
$$

Setting $w=u^{(1)}-u^{(2)}$, we obtain

$$
\frac{w_{j}}{\tau}+\frac{1}{2}(v+i \eta) \Delta_{h}^{\alpha} w_{j}+(\kappa+i \zeta) g_{j}-\frac{1}{2} \gamma w_{j}=0, \quad j \in \mathscr{T}_{M},
$$

where

$$
g_{j}=\left|\frac{u_{j}^{(1)}+u_{j}^{n}}{2}\right|^{2} \frac{u_{j}^{(1)}+u_{j}^{n}}{2}-\left|\frac{u_{j}^{(2)}+u_{j}^{n}}{2}\right|^{2} \frac{u_{j}^{(2)}+u_{j}^{n}}{2} .
$$

Computing the discrete inner product of (4.27) with $w$ and taking the real part give

$$
\|w\|_{h}^{2}+\frac{v \tau}{2}\left\|\Lambda^{\alpha} w\right\|_{h}^{2}+\tau \operatorname{Re}(\kappa+i \zeta)(g, w)_{h}=\frac{\gamma \tau}{2}\|w\|_{h}^{2} .
$$

For the third term on the left-hand side of (4.28), invoking (4.26), we have

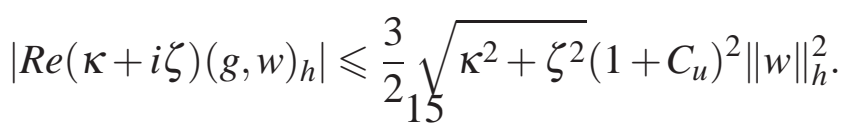


Substituting above inequality into (4.28) yields

$$
\|w\|_{h}^{2} \leqslant \tau \frac{3 \sqrt{\kappa^{2}+\zeta^{2}}\left(1+C_{u}\right)^{2}+|\gamma|}{2}\|w\|_{h}^{2}
$$

For $\tau<\frac{2}{3 \sqrt{\kappa^{2}+\zeta^{2}}\left(1+C_{u}\right)^{2}+|\gamma|}$, we obtain $\|w\|_{h}=0$, which implies

$$
w_{j}=0, \quad j \in \mathscr{T}_{M} \text {. }
$$

Thus, the proof is complete.

\section{Numerical experiments}

In this section, we report some numerical results to confirm the theoretical accuracy and efficiency of scheme (3.7)-(3.9).

\subsection{Iterative algorithm}

Before embarking on our numerical experiments, developing an efficient iterative algorithm is of the essence to compute the solution of the system of nonlinear equation arising at a given time level of the scheme (3.7)-(3.9).

To this end, rewrite (3.7) as

$$
u_{j}^{n+\frac{1}{2}}=u_{j}^{n}-\frac{\tau}{2}\left[(v+i \eta) \Delta_{h}^{\alpha} u_{j}^{n+\frac{1}{2}}+(\kappa+i \zeta)\left|u_{j}^{n+\frac{1}{2}}\right|^{2} u_{j}^{n+\frac{1}{2}}-\gamma u_{j}^{n+\frac{1}{2}}\right] .
$$

We introduce the variable $z_{j}=u_{j}^{n+\frac{1}{2}}$ and then obtain

$$
z_{j}=u_{j}^{n}-\frac{\tau}{2}\left[(v+i \eta) \Delta_{h}^{\alpha} z_{j}+(\kappa+i \zeta)\left|z_{j}\right|^{2} z_{j}-\gamma z_{j}\right],
$$

and

$$
u_{j}^{n+1}=2 z_{j}-u_{j}^{n} .
$$

Then we propose the following iterative algorithm

$$
\begin{gathered}
z_{j}^{(s+1)}=u_{j}^{n}-\frac{\tau}{2}\left[(v+i \eta) \Delta_{h}^{\alpha} z_{j}^{(s+1)}+(\kappa+i \zeta)\left|z_{j}^{(s)}\right|^{2} z_{j}^{(s)}-\gamma z_{j}^{(s+1)}\right], \\
j \in \mathscr{T}_{M}, 0 \leqslant n \leqslant N-1, s=0,1,2, \ldots, \\
z_{j}^{(s)}=0, \quad j \in \mathbb{Z} \backslash \mathscr{T}_{M}, \quad 0 \leqslant n \leqslant N, s=0,1,2, \ldots
\end{gathered}
$$

The initial iteration is selected as, for $n \geqslant 1$,

$$
z_{j}^{(0)}=\frac{3}{2} u_{j}^{n}-\frac{1}{2} u_{j}^{n-1}
$$

and for $n=0$,

$$
z_{j}^{(0)}=u_{j}^{0}-\frac{\tau}{2}\left[(v+i \eta) \Delta_{h}^{\alpha} u_{j}^{0}+(\kappa+i \zeta)\left|u_{j}^{0}\right|^{2} u_{j}^{0}-\gamma u_{j}^{0}\right] .
$$

The system is indeed linearized at each iteration, and we solve an inner problem $A z^{(s+1)}=b$ to get $z_{j}^{(s+1)}$. Then $z_{j}$ is numerically reached once $z_{j}^{(s+1)}$ converges and $u_{j}^{n+1}$ is obtained by (5.3). It is noted that the coefficient matrix $A$ is independent of the time level, and this feature dramatically benefits the numerical implementation. 


\subsection{Numerical tests}

Example 5.2.1 We firstly testify the numerical accuracy of the scheme with

$$
v=0.3, \eta=\frac{1}{2}, \kappa=-\frac{v\left(3 \sqrt{1+4 v^{2}}-1\right)}{2\left(2+9 v^{2}\right)}, \zeta=-1, \gamma=0
$$

For $\alpha=2$, the exact solution is explicitly given by [43]

$$
u(x, t)=a(x) \exp (i d \ln [a(x)]-i \omega t)
$$

where

$$
a(x)=F \operatorname{sech}(x), F=\sqrt{\frac{d \sqrt{1+4 v^{2}}}{-2 \kappa}}, d=\frac{\sqrt{1+4 v^{2}}-1}{2 v}, \omega=-\frac{d\left(1+4 v^{2}\right)}{2 v} .
$$

The computational interval is chosen as $[a, b]=[-16,16]$ and the initial value is taken as $u(x, 0)$ in (5.5). Choose the iteration tolerance as $10^{-14}$ for the iterative algorithm (5.4). For $1<\alpha<2$, the exact solution can not be explicitly given and thus, the numerical "exact" solution $u$ is computed using the proposed scheme with a very fine mesh size $h=0.0125$ and time step $\tau=0.0001$. Let $u_{h}$ be the numerical solution. We measure the error $e(\tau, h)=u-u_{h}$ at time $T=1$ with the $l^{2}$ norm and the $l^{\infty}$ norm. The corresponding convergence orders are calculated by

$$
\begin{aligned}
& \text { order1 }=\log _{2}\left(\|e(h, \tau)\|_{h} /\|e(\tau / 2, h / 2)\|_{h}\right), \\
& \text { order2 }=\log _{2}\left(\|e(h, \tau)\|_{l_{h}^{\infty}} /\|e(\tau / 2, h / 2)\|_{l_{h}^{\infty}}\right) .
\end{aligned}
$$

Tables 1, 2 list the errors and corresponding orders of the numerical scheme for $\alpha=2$ and $1<$ $\alpha<2$, respectively. The data confirm the theoretical accuracy of the difference scheme (3.7)-(3.9) in Theorem 4.3

Table 1: The $l_{h}^{2}$ and $l_{h}^{\infty}$ errors and their convergence orders for $\alpha=2$.

\begin{tabular}{llllll}
\hline$\tau$ & $h$ & $\|e\|_{h}$ & order1 & $\|e\|_{l_{h}^{\infty}}$ & order2 \\
\hline 0.02 & 0.2 & $5.5462 \mathrm{e}-003$ & - & $5.5486 \mathrm{e}-003$ & - \\
0.01 & 0.1 & $1.3766 \mathrm{e}-003$ & 2.0104 & $1.3691 \mathrm{e}-003$ & 2.0190 \\
0.005 & 0.05 & $3.4353 \mathrm{e}-004$ & 2.0026 & $3.4117 \mathrm{e}-004$ & 2.0046 \\
0.0025 & 0.025 & $8.5845 \mathrm{e}-005$ & 2.0006 & $8.5225 \mathrm{e}-005$ & 2.0011 \\
0.00125 & 0.0125 & $2.1460 \mathrm{e}-005$ & 2.0001 & $2.1302 \mathrm{e}-005$ & 2.0003 \\
\hline
\end{tabular}

Example 5.2.2 In this tests, the initial value is chosen as

$$
u(x, 0)=\exp _{17}\left(-2 x^{2}\right) .
$$


Table 2: The $l_{h}^{2}$ and $l_{h}^{\infty}$ errors and their convergence orders for $1<\alpha<2$.

\begin{tabular}{lllllll}
\hline$\alpha$ & $h$ & $\tau$ & $\|e\|_{h}$ & order1 & $\|e\|_{l_{h}^{\infty}}$ & order2 \\
\hline \multirow{4}{*}{1.3} & 0.2 & 0.02 & $1.2966 \mathrm{e}-002$ & - & $1.8415 \mathrm{e}-002$ & - \\
& 0.1 & 0.01 & $3.1803 \mathrm{e}-003$ & 2.0275 & $4.4581 \mathrm{e}-003$ & 2.0464 \\
& 0.05 & 0.005 & $7.5488 \mathrm{e}-004$ & 2.0749 & $1.0528 \mathrm{e}-003$ & 2.0822 \\
\hline \multirow{4}{*}{1.6} & 0.2 & 0.02 & $1.0519 \mathrm{e}-002$ & - & $1.3001 \mathrm{e}-002$ & - \\
& 0.1 & 0.01 & $2.5499 \mathrm{e}-003$ & 2.0444 & $3.0928 \mathrm{e}-003$ & 2.0717 \\
& 0.05 & 0.005 & $6.0393 \mathrm{e}-004$ & 2.0780 & $7.2919 \mathrm{e}-004$ & 2.0846 \\
\hline \multirow{3}{*}{1.9} & 0.2 & 0.02 & $6.7430 \mathrm{e}-003$ & - & $7.0782 \mathrm{e}-003$ & - \\
& 0.1 & 0.01 & $1.6458 \mathrm{e}-003$ & 2.0346 & $1.7127 \mathrm{e}-003$ & 2.0471 \\
& 0.05 & 0.005 & $3.9052 \mathrm{e}-004$ & 2.0753 & $4.0552 \mathrm{e}-004$ & 2.0784 \\
\hline
\end{tabular}

Henceforth, we always take $[a, b]=[-10,10]$ and $\tau=h=0.05$.

Firstly, the evolution of the numerical solution is depicted. We pay particular attention to the influence of parameter $\gamma$ on the evolution of wave-shape in the fractional case. To this end, chosen $v=1, \eta=1, \kappa=1, \zeta=2$, we take different values of $\gamma$, i.e., $\gamma=2,1,0,-1,-2$ with $\alpha=1.8$ and depict the evolution of $|u|$ in Figures 1, 3. It is observed that, as in the classical case, the parameter $\gamma$ dramatically affects the wave-shape in the fractional case. In addition, the solution decays rapidly with time evolution especially for $\gamma<0$. For more intensive study, then in Figure 4 , we further depict the evolution of $\|u\|_{h}^{2}$ with $\alpha=1.3,1.8$. Recall that, in the classical case $(\alpha=2)$, the discrete norm $\|u\|_{h}^{2}$ decays to zero for $\gamma<0$ and when $\gamma$ is smaller, the decay is faster [18]. In our fractional case, we observe similar phenomena and the fractional order $\alpha$ affects the decay rate very slightly.

Secondly, we numerically study the impact on the dissipative mechanism of the fractional Laplacian. Choose $v=1, \eta=1, \kappa=1, \zeta=2, \gamma=3$. The solutions at $t=1$ with different values of $\alpha$ are depicted in Figure 5. It can be seen that the wave-shape changes with fractional parameter $\alpha$. This phenomenon is greatly different from that in the classical case and essentially, features the nonlocal character of the fractional Laplacian.

Finally, we simulate the inviscid limit behavior of the solution. The authors in [11] have shown that the solution of the FGLE converges to the solution of the FSE (i.e., $v=0, \kappa=0$ ) when $v \rightarrow 0, \kappa \rightarrow 0$. For the numerical simulation of the FSE, see, e.g., [41, 44, 45, 46]. Here we numerically testify this matter. For this purpose, we set $\eta=1, \zeta=-2, \gamma=0$ and choose diminishing $v$ and $\kappa$. From Figure 6 , it is observed that the solution asymptotically approachs to the solution of the FSE. This observation confirms the theoretical results in [11]. 

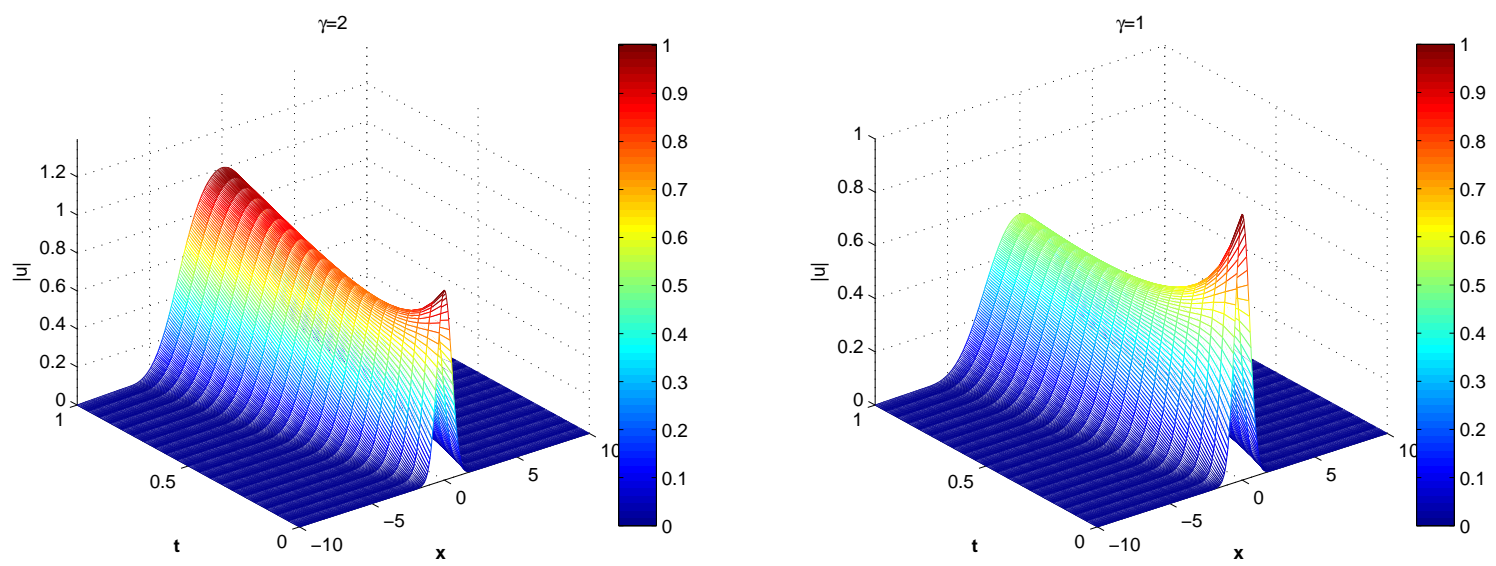

Figure 1: The evolution of $|u|$ for $\gamma=2$ (left) and $\gamma=1$ (right).
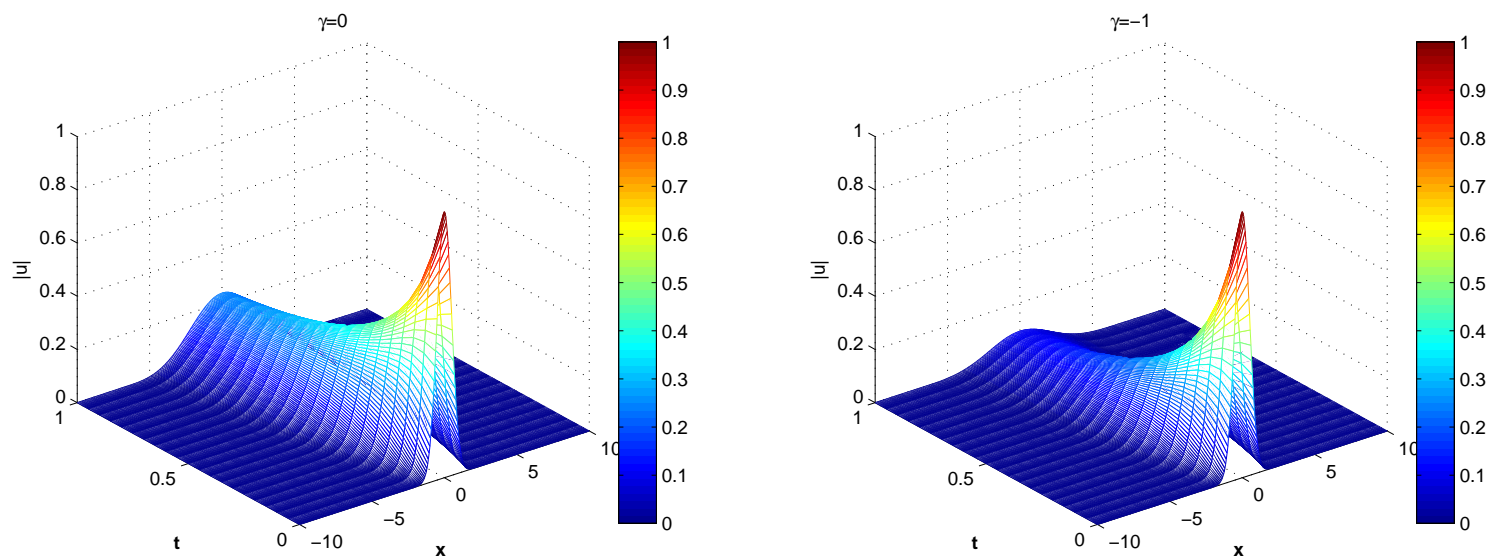

Figure 2: The evolution of $|u|$ for $\gamma=0$ (left) and $\gamma=-1$ (right).

\section{Conclusions}

In this paper, we proposed and analyzed a finite difference scheme for solving the nonlinear complex fractional Ginzburg-Landau equation where the fractional Laplacian was approximated by the weighted and shifted Grünwald difference operator. We obtained the unconditional optimal convergence rate at the order of $O\left(\tau^{2}+h^{2}\right)$ in the $l_{h}^{2}$ norm with the time step $\tau$ and mesh size $h$, without any mesh ratio constraints. In the proof for the scheme, building on the careful analysis of the difference operator, we established some useful inequalities with respect to the fractional Sobolev norm and the a priori bound of the numerical solution. Both theoretical analysis and numerical tests show that the scheme is efficient for the numerical solution of the nonlinear fractional Ginzburg-Landau equation. 


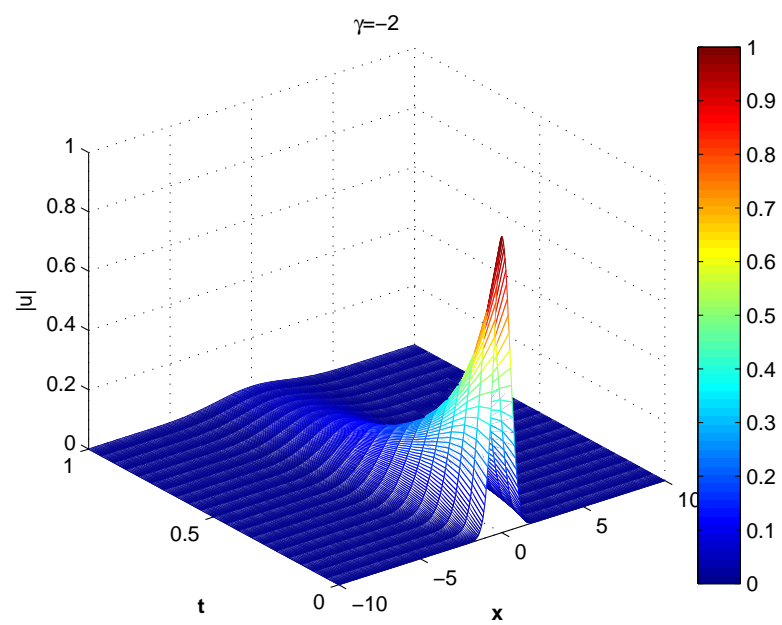

Figure 3: The evolution of $|u|$ for $\gamma=-2$.
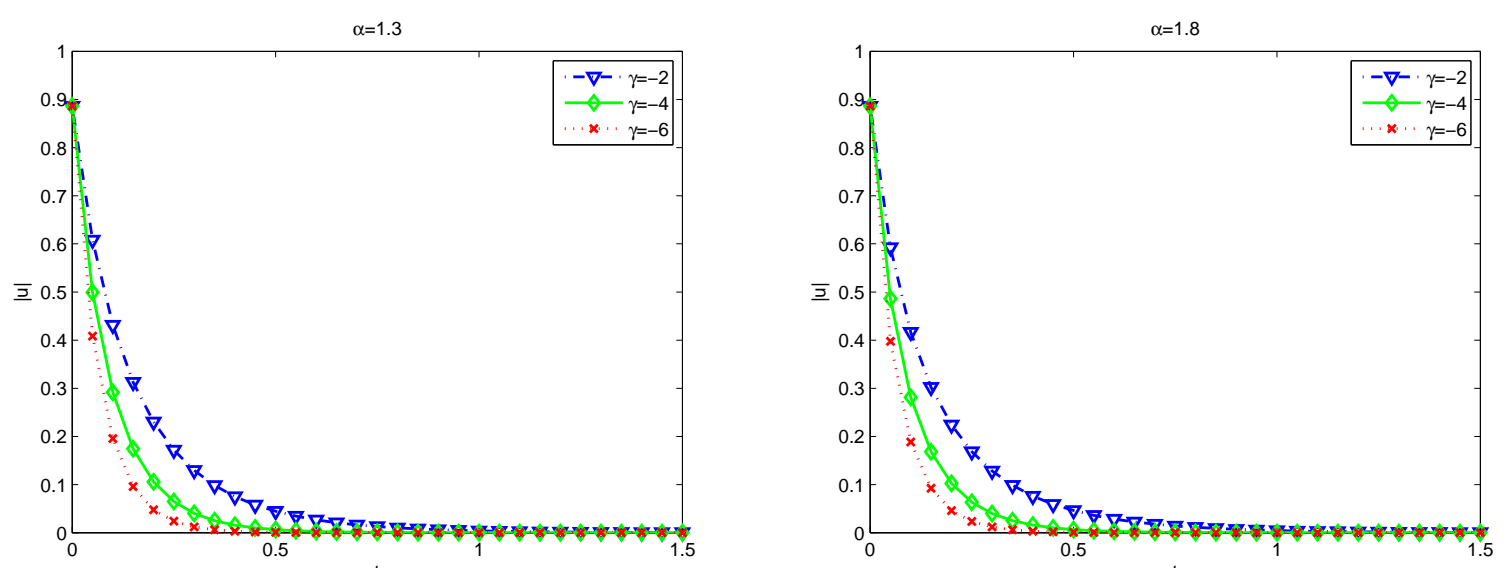

Figure 4: The discrete norm $\|u\|_{h}^{2}$ at for $\alpha=1.3$ (left) and $\alpha=1.8$ (right) with $\gamma=-2,-4,-6$.

\section{Acknowledgments}

This work was supported by National Natural Science Foundation of China (Nos. 91130003 and 11371157) and the Graduates' Innovation Fund of Huazhong University of Science \& Technology (No. 2015650011). The authors wish to thank the anonymous referees for their valuable comments and suggestions which lead to an improvement of this paper.

\section{Appendix A: Hausdorff-Young inequality}

Lemma A.1. If $1 \leqslant q \leqslant 2, \frac{1}{q}+\frac{1}{p}=1$, then

$$
\left(h \sum_{j \in \mathbb{Z}}\left|u_{j}\right|^{p}\right)^{\frac{1}{p}} \leqslant C\left(\int_{-\pi / h}^{\pi / h}|\widehat{u}(k)|^{q} \mathrm{~d} k\right)^{\frac{1}{q}},
$$




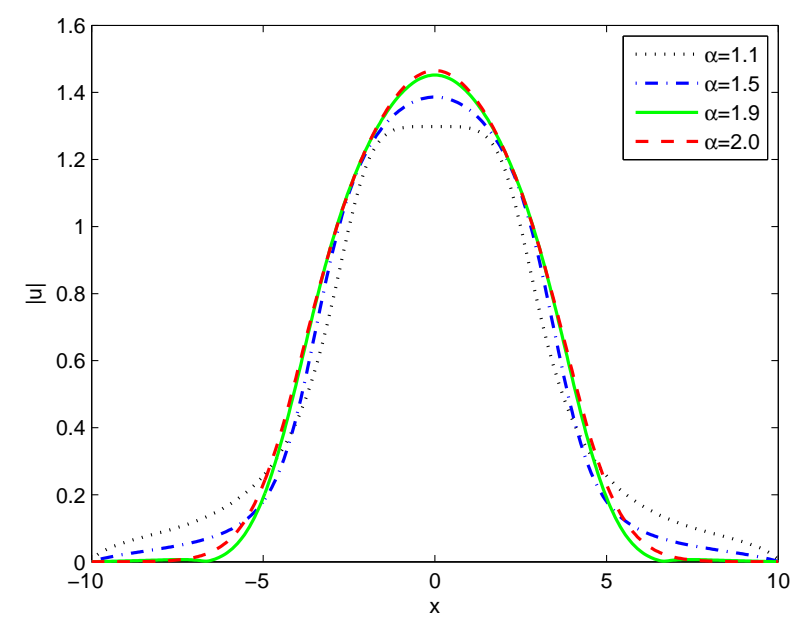

Figure 5: The profile of $|u|$ at $t=1$ with $\alpha=1.1,1.5,1.9,2.0$.
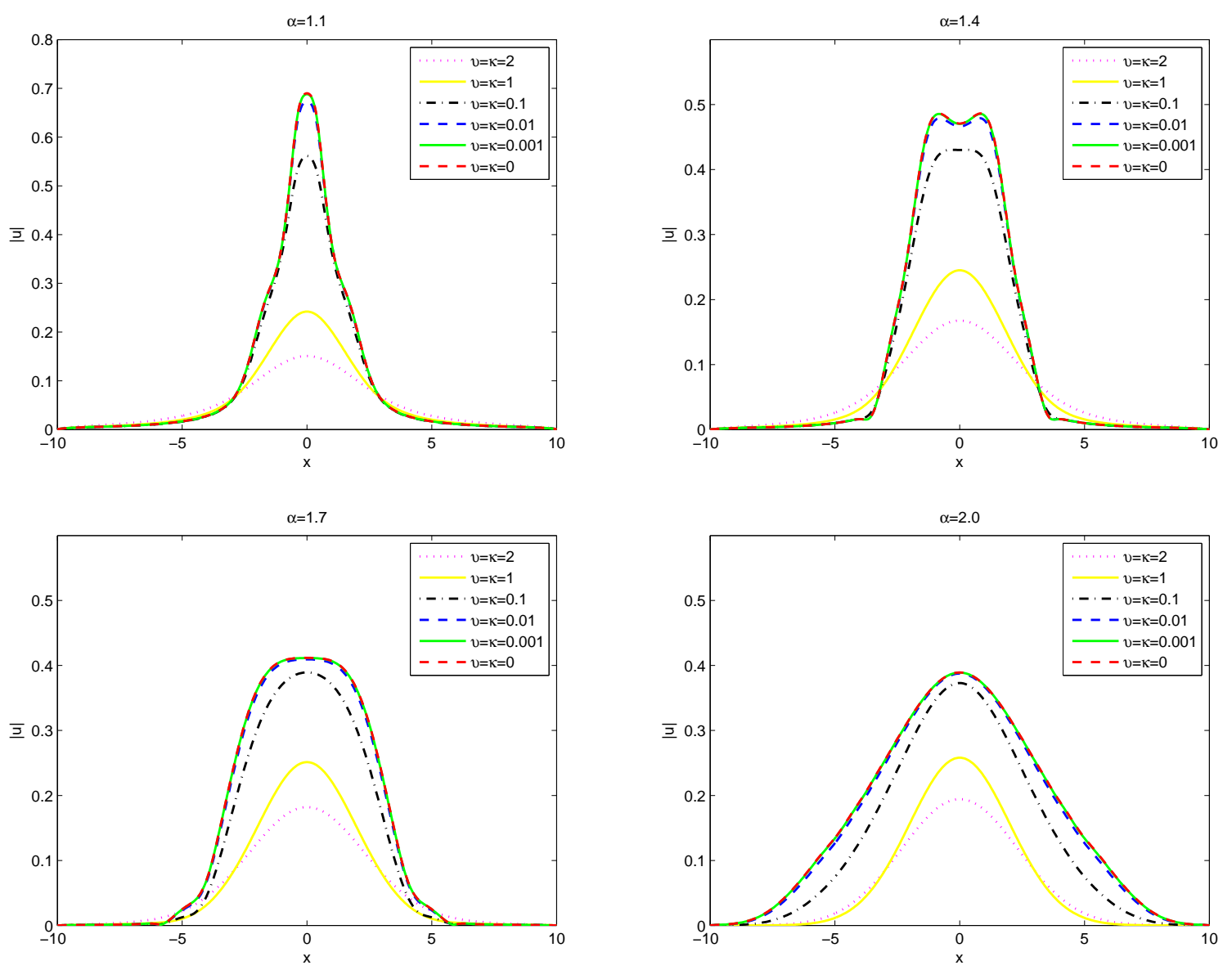

Figure 6: The profile of $|u|$ at $t=1$ with diminishing $v$ and $\kappa$ for $\alpha=1.1,1.4,1.7,2.0$. 
where $\frac{1}{\sqrt{2 \pi}} \leqslant C \leqslant 1$.

Proof. The proof is very similar to that of the same inequality for Fourier series (see Corollary 2.4 in [47, page 57]) and hence, we just give below a sketch of it. From the definition of inversion formula, we have

$$
\sup _{j \in \mathbb{Z}}\left|u_{j}\right| \leqslant \frac{1}{\sqrt{2 \pi}} \int_{-\pi / h}^{\pi / h}|\widehat{u}(k)| \mathrm{d} k .
$$

The Parseval's indentity gives

$$
h \sum_{j \in \mathbb{Z}}\left|u_{j}\right|^{2}=\int_{-\pi / h}^{\pi / h}|\widehat{u}(k)|^{2} \mathrm{~d} k .
$$

Then invoking the Riesz interpolation theorem (see Theorem 2.1 in [47, page 52]), we obtain the conclusion.

\section{Appendix B: The proof of Lemma 2.4}

Lemma 2.4, For $1<\alpha \leqslant 2$, let $h(\alpha, \omega)$ be the function defined by

$$
h(\alpha, \omega)=\lambda_{1} \cos \left(\frac{\alpha}{2}(\omega-\pi)-\omega\right)+\lambda_{0} \cos \left(\frac{\alpha}{2}(\omega-\pi)\right)+\lambda_{-1} \cos \left(\frac{\alpha}{2}(\omega-\pi)+\omega\right),
$$

where $\omega \in[0, \pi]$ and $\lambda_{1}, \lambda_{0}, \lambda_{-1}$ are defined in (2.4). Then $h(\alpha, \omega)$ does not decrease with respect to $\omega$.

Proof. It is easy to see that $h(\alpha, \omega) \equiv-1$ for $\alpha=2$.

For $1<\alpha<2$, rearranging $h(\alpha, \omega)$ gives

$$
\begin{aligned}
h(\alpha, \omega)= & \frac{\alpha^{2}+3 \alpha+2}{12} \cos \left(\frac{\alpha}{2}(\omega-\pi)-\omega\right)+\frac{4-\alpha^{2}}{6} \cos \left(\frac{\alpha}{2}(\omega-\pi)\right) \\
& +\frac{\alpha^{2}-3 \alpha+2}{12} \cos \left(\frac{\alpha}{2}(\omega-\pi)+\omega\right) \\
= & \frac{\alpha^{2}+2}{12}\left(\cos \left(\frac{\alpha}{2}(\omega-\pi)-\omega\right)+\cos \left(\frac{\alpha}{2}(\omega-\pi)+\omega\right)\right) \\
& +\frac{3 \alpha}{12}\left(\cos \left(\frac{\alpha}{2}(\omega-\pi)-\omega\right)-\cos \left(\frac{\alpha}{2}(\omega-\pi)+\omega\right)\right)+\frac{4-\alpha^{2}}{6} \cos \left(\frac{\alpha}{2}(\omega-\pi)\right) \\
= & \frac{\alpha^{2}+2}{6} \cos \left(\frac{\alpha}{2}(\omega-\pi)\right) \cos (\omega)+\frac{\alpha}{2} \sin \left(\frac{\alpha}{2}(\omega-\pi)\right) \sin (\omega)+\frac{4-\alpha^{2}}{6} \cos \left(\frac{\alpha}{2}(\omega-\pi)\right) .
\end{aligned}
$$


Taking the derivative of $h(\alpha, \omega)$ with respect to $\omega$, we obtain

$$
\begin{aligned}
h^{\prime}(\alpha, \omega)= & \frac{\alpha^{2}+2}{6}\left(-\frac{\alpha}{2} \sin \left(\frac{\alpha}{2}(\omega-\pi)\right) \cos (\omega)-\cos \left(\frac{\alpha}{2}(\omega-\pi)\right) \sin (\omega)\right) \\
& +\frac{\alpha}{2}\left(\frac{\alpha}{2} \cos \left(\frac{\alpha}{2}(\omega-\pi)\right) \sin (\omega)+\sin \left(\frac{\alpha}{2}(\omega-\pi)\right) \cos (k)\right)-\frac{\alpha\left(4-\alpha^{2}\right)}{12} \sin \left(\frac{\alpha}{2}(\omega-\pi)\right) \\
= & \frac{\alpha\left(4-\alpha^{2}\right)}{12} \sin \left(\frac{\alpha}{2}(\omega-\pi)\right)(\cos (\omega)-1)-\frac{4-\alpha^{2}}{12} \cos \left(\frac{\alpha}{2}(\omega-\pi)\right) \sin (\omega) \\
= & -\frac{\alpha\left(4-\alpha^{2}\right)}{6} \sin \left(\frac{\alpha}{2}(\omega-\pi)\right) \sin ^{2}\left(\frac{\omega}{2}\right)-\frac{4-\alpha^{2}}{6} \cos \left(\frac{\alpha}{2}(\omega-\pi)\right) \sin \left(\frac{\omega}{2}\right) \cos \left(\frac{\omega}{2}\right) \\
= & -\frac{4-\alpha^{2}}{6} \sin \left(\frac{\omega}{2}\right) g(\alpha, \omega),
\end{aligned}
$$

where

$$
g(\alpha, \omega):=\alpha \sin \left(\frac{\alpha}{2}(\omega-\pi)\right) \sin \left(\frac{\omega}{2}\right)+\cos \left(\frac{\alpha}{2}(\omega-\pi)\right) \cos \left(\frac{\omega}{2}\right) .
$$

In order to show that $h(\alpha, \omega)$ non-decreases with respect to $\omega$, it is sufficient to show that $h^{\prime}(\alpha, \omega) \geqslant$ 0 , or $g(\alpha, \omega) \leqslant 0$ for $\omega \in[0, \pi]$. In fact, taking the derivative of $g(\alpha, \omega)$ with respect to $\omega$ yields

$$
g^{\prime}(\alpha, \omega)=\frac{\alpha^{2}-1}{2} \cos \left(\frac{\alpha}{2}(\omega-\pi)\right) \sin \left(\frac{\omega}{2}\right),
$$

which implies that the extreme point of $g(\alpha, \omega)$ is $\omega=\frac{\alpha-1}{\alpha} \pi$ for $\omega \in(0, \pi)$. By a simple analysis, it is shown that $g(\alpha, \omega)$ reach its maximum value at $\omega=\pi$ and reach its minimum value at $\omega=$ $\frac{\alpha-1}{\alpha} \pi$, which gives

$$
-\alpha \sin \left(\frac{\alpha-1}{2 \alpha} \pi\right)=g\left(\alpha, \frac{\alpha-1}{\alpha} \pi\right) \leqslant g(\alpha, \omega) \leqslant g(\alpha, \pi)=0 .
$$

Thus, the proof is complete.

\section{References}

[1] I. S. Aranson, L. Kramer, The world of the complex Ginzburg-Landau equation, Rev. Mod. Phys. 74 (2002) 99-143.

[2] V. Tarasov, G. Zaslavsky, Fractional Ginzburg-Landau equation for fractal media, Phys. A 354 (2005) $249-261$.

[3] V. Tarasov, G. Zaslavsky, Fractional dynamics of coupled oscillators with long-range interaction, Chaos 16 (2006) 023110.

[4] A. Milovanov, J. Rasmussen, Fractional generalization of the Ginzburg-Landau equation: an unconventional approach to critical phenomena in complex media, Phys. Lett. A 337 (2005) 75-80.

[5] A. Mvogo, A. Tambue, G. Ben-Bolie, T. Kofane, Localized numerical impulses solutions in diffuse neural networks modeled by the complex fractional Ginzburg-Landau equation (2014) arXiv:1411.7983.

[6] P. Zhuang, F. Liu, V. Anh, I. Turner, Numerical methods for the variable-order fractional advection-diffusion equation with a nonlinear source term, SIAM J. Numer. Anal. 47 (2009) 1760-1781.

[7] Q. Yang, F. Liu, I. Turner, Numerical methods for fractional partial differential equations with Riesz space fractional derivatives, Appl. Math. Model. 34 (2010) 200-218.

[8] I. Podlubny, Fractional Differential Equations, Academic Press, San Diego, 1999. 
[9] V. Tarasov, Psi-series solution of fractional Ginzburg-Landau equation, J. Phys. A: Math. Gen. 39 (2006) 8395-8407.

[10] X. Pu, B. Guo, Well-posedness and dynamics for the fractional Ginzburg-Landau equation, Appl. Anal. 92 (2013) 318-334.

[11] B. Guo, Z. Huo, Well-posedness for the nonlinear fractional Schrödinger equation and inviscid limit behavior of solution for the fractional Ginzburg-Landau equation, Fract. Calc. Appl. Anal. 16 (2013) 226-242.

[12] H. Lu, S. Lü, Z. Feng, Asymptotic dynamics of 2D fractional complex Ginzburg-Landau equation, Int. J. Bifurcation Chaos 13 (2013) 1350202.

[13] V. Millot, Y. Sire, On a fractional Ginzburg-Landau equation and 1/2-harmonic maps into spheres, Arch. Rational Mech. Anal. 215 (2015) 125-210.

[14] Z. P. Hao, Z. Z. Sun, W. R. Cao, A fourth-order approximation of fractional derivatives with its applications, J. Comput. Phys. 281 (2015) 787-805.

[15] G. J. Lord, Attractors and inertial manifolds for finite-difference approximations of the complex GinzburgLandau equation, SIAM J.Numer. Anal. 34 (1997) 1483-1512.

[16] Z. Z. Sun, Q. Zhu, On Tsertsvadze's difference scheme for the Kuramoto-Tsuzuki equation, J. Comput. Appl. Math. 98 (1998) 289-304.

[17] T. Matsuo, D. Furihatay, Dissipative or conservative finite-difference schemes for complex-valued nonlinear partial differential equations, J. Comput. Phys. 171 (2001) 425-447.

[18] Q. Xu, Q. Chang, Difference methods for computing the Ginzburg-Landau equation in two dimensions, Numer. Methods Partial Differ. Equ. 27 (2011) 507-528.

[19] T. Wang, B. Guo, Analysis of some finite difference schemes for two-dimensional Ginzburg-Landau equation, Numer. Methods Partial Differ. Equ. 27 (2011) 1340-1363.

[20] L. Zhang, Long time behavior of difference approximations for the two-dimensional complex Ginzburg-Landau equation, Numer. Funct. Anal. Optim. 31 (2010) 1190-1211.

[21] Y. N. Zhang, Z. Z. Sun, T. Wang, Convergence analysis of a linearized Crank-Nicolson scheme for the twodimensional complex Ginzburg-Landau equation, Numer. Methods Partial Differ. Equ. 29 (2013) 1487-1503.

[22] Z. P. Hao, Z. Z. Sun, W. R. Cao, A three-level linearized compact difference scheme for the Ginzburg-Landau equation, Numer. Methods Partial Differ. Equ. 31 (2015) 876-899.

[23] M. M. Meerschaert, C. Tadjeran, Finite difference approximations for fractional advection-dispersion flow equations, J. Comput. Appl. Math. 172 (2004) 65-77.

[24] C. Tadjeran, M. M. Meerschaert, H. P. Scheffler, A second-order accurate numerical approximation for the fractional diffusion equation, J. Comput. Phys. 213 (2006) 205-213.

[25] W. Tian, H. Zhou, W. Deng, A class of second order difference approximation for solving space fractional diffusion equations, Math. Comp. 84 (2015) 1703-1727.

[26] H. Zhou, W. Tian, W. Deng, Quasi-compact finite difference schemes for space fractional diffusion equations, J. Sci. Comput. 56 (2013) 45-66.

[27] M. Chen, W. Deng, Fourth order accurate scheme for the space fractional diffusion equations, SIAM J. Numer. Anal. 52 (2014) 1418-1438.

[28] M. Chen, W. Deng, Fourth order difference approximations for space Riemann-Liouville derivatives based on weighted and shifted Lubich difference operators, Commun. Comput. Phys. 16 (2014) 516-540.

[29] B. Baeumer, M. Kovács, H. Sankaranarayanan, Higher order Grünwald approximations of fractional derivatives and fractional powers of operators, Trans. Amer. Math. Soc. 367 (2015) 813-834.

[30] L. Zhao, W. Deng, A series of high-order quasi-compact schemes for space fractional diffusion equations based on the superconvergent approximations for fractional derivatives, Numer. Methods Partial Differ. Equ. 31 (2015) 1345-1381.

[31] M. D. Ortigueira, Riesz potential operators and inverses via fractional centred derivatives, Int. J. Math. Math. Sci. (2006) 1-12.

[32] C. Çelik, M. Duman, Crank-Nicolson method for the fractional diffusion equation with the Riesz fractional derivative, J. Comput. Phys. 231 (2012) 1743-1750.

[33] H. Ding, C. Li, Y. Chen, High-order algorithms for Riesz derivative and their applications (II), J. Comput. Phys. 293 (2015) 218-237. 
[34] X. Zhao, Z. Z. Sun, Z. P. Hao, A fourth-order compact ADI scheme for two-dimensional nonlinear space fractional Schrödinger equation, SIAM J. Sci. Comput. 36 (2014) A2865-A2886.

[35] L. N. Trefethen, Spectral methods in MATLAB, SIAM, Philadelphia, 2000.

[36] J. C. Strikwerda, Finite difference schemes and partial differential equations, 2nd ed., SIAM, Philadelphia, 2004.

[37] K. Kirkpatrick, E. Lenzmann, G. Staffilani, On the continuum limit for discrete NLS with long-range lattice interactions, Commun. Math. Phys. 317 (2013) 563-591.

[38] Q. Du, M. Gunzburger, R. B. Lehoucq, K. Zhou, Analysis and approximation of nonlocal diffusion problems with volume constraints, SIAM Rev. 54 (2012) 667-696.

[39] O. Defterli, M. D’Elia, Q. Du, M. Gunzburger, R. Lehoucq, M. M. Meerschaert, Fractional diffusion on bounded domains, Fract. Calc. Appl. Anal. 18 (2015) 342-360.

[40] R. A. Horn, C. R. Johnson, Matrix Analysis, Cambridge Univ. Press, 1986.

[41] P. Wang, C. Huang, An energy conservative difference scheme for the nonlinear fractional Schrödinger equations, J. Comput. Phys. 293 (2015) 238-251.

[42] G. D. Akrivis, V. A. Dougalis, O. A. Karakashian, On fully discrete Galerkin methods of second-order temporal accuracy for the nonlinear Schrödinger equation, Numer. Math. 59 (1991) 31-53.

[43] N. N. Akhmediev, V. V. Afanasjev, J. M. Soto-Crespo, Singularities and special soliton solutions of the cubicquintic complex Ginzburg-Landau equation, Phys. Rev. E 53 (1996) 1190-1201.

[44] P. Wang, C. Huang, A conservative linearized difference scheme for the nonlinear fractional Schrödinger equation, Numer. Algor. 69 (2015) 625-641.

[45] D. Wang, A. Xiao, W. Yang, Crank-Nicolson difference scheme for the coupled nonlinear Schrödinger equations with the Riesz space fractional derivative, J. Comput. Phys. 242 (2013) 670-681.

[46] D. Wang, A. Xiao, W. Yang, A linearly implicit conservative difference scheme for the space fractional coupled nonlinear Schrödinger equations, J. Comput. Phys. 272 (2014) 644-655.

[47] E. M. Stein, R. Shakarchi, Functional analysis: introduction to further topics in analysis, Princeton Lectures in Analysis IV, Princeton University Press, 2011. 